\title{
New families of self-dual codes
}

\author{
Lin Sok*
}

\begin{abstract}
In the recent paper entitled "Explicit constructions of MDS selfdual codes" accepted in IEEE Transactions on Information Theory, doi: 10.1109/TIT.2019.2954877, the author has constructed families of MDS self-dual codes from genus zero algebraic geometry (AG) codes, where the AG codes of length $n$ were defined using two divisors $G$ and $D=P_{1}+\cdots+P_{n}$. In the present correspondence, we explore more families of optimal self-dual codes from AG codes. New families of MDS self-dual codes with odd characteristics and those of almost MDS self-dual codes are constructed explicitly from genus zero and genus one curves, respectively. More families of self-dual codes are constructed from algebraic curves of higher genus.
\end{abstract}

Keywords: Self-orthogonal codes, self-dual codes, MDS codes, almost MDS codes, optimal codes, algebraic curves, algebraic geometry codes, differential algebraic geometry codes

\section{Introduction}

Self-dual codes are one of the most interesting classes of linear codes that find diverse applications in cryptographic protocols (secret sharing schemes) introduced in [4, 5, 18] and combinatorics [17]. It is well-known that binary self-dual codes are asymptotically good [16].

MDS codes form an optimal family of classical codes. They are closely related to combinatorial designs [17, p. 328], and finite geometries [17, p.

*School of Mathematical Sciences, Anhui University, Hefei, Anhui, 230601, soklin_heng@yahoo.com 
326]. Due to their largest error correcting capability for given length and dimension, MDS codes are of great interest in both theory and practice. The most well-known family of MDS linear codes is that of Reed-Solomon codes. MDS linear codes exist in a very restrict condition on their lengths as the famous MDS conjecture states: for every linear $[n, k, n-k+1]$ MDS code over $\mathbb{F}_{q}$, if $1<k<q$, then $n \leq q+1$, except when $q$ is even and $k=3$ or $k=q-1$, in which cases $n \leq q+2$. The conjecture was proved by Ball [1] for $q$ a prime. However, for self-dual case, the conjecture may not be true.

Due to the reasons mentioned above, MDS self-dual codes have been of much interest to many researchers. As we have already known that determining the parameters of a given linear code is a challenging problem in coding theory. However, the parameters of an MDS self-dual code are completely determined by its length. Constructions of MDS self-dual codes are valuable. For classical constructions of MDS self-dual codes, we refer to [2, 8, 14, 11]. Existing families of MDS self-dual codes can be described as follows. Grassl et al. [10] constructed MDS codes of all lengths over $\mathbb{F}_{2^{m}}$ and of all highest possible length over finite fields of odd characteristics. Jin et al. [13] proved the existence of MDS self-dual codes over $\mathbb{F}_{q}$ in odd characteristic for $q \equiv 1$ $(\bmod 4)$ and for $q$ a square of a prime for some restricted lengths. Using the same technique developed in [13, more families of MDS self-dual codes have been constructed in [24, 7]. Tong et al. 223] gave constructions of MDS Euclidean self-dual codes through cyclic duadic codes. The families of known MDS self-dual codes are summarized in Table 1.

The discovery of algebraic geometry codes in 1981 was due to Goppa [9], where they were also called geometric Goppa codes. Goppa showed in his paper [9] how to construct linear codes from algebraic curves over a finite field. Despite a strongly theoritical construction, algebraic geometry (AG) codes have asymptotically good parameters, and it was the first time that linear codes improved the so-called Gilbert-Vasharmov bound. Self-dual AG codes were studied by Stichtenoth [20] and Driencourt et al. [6], where they first characterized such codes. However, the construction of MDS self-dual AG codes with odd characteristics or almost MDS self-dual AG codes was not considered there.

On the contrary to the MDS case, almost MDS codes exist more frequently, and it is thus worth exploring families of self-dual codes in such a case and those of optimal self-dual codes. In [20], Stichtenoth gave constructions of self-orthogonal AG codes (and self-dual AG codes for some special cases) but did not consider an embedding the self-orthogonal codes into the 
Table 1: Existing families of MDS self-dual codes, $\eta$ : the quadratic character of $\mathbb{F}_{q}$

\begin{tabular}{|c|c|c|}
\hline$q$ & $n$ & References \\
\hline $\begin{aligned} q & =2^{m} \\
q & =p^{m}, p \text { odd prime }\end{aligned}$ & $\begin{array}{l}n \leq q \\
n=q+1\end{array}$ & 10 \\
\hline $\begin{array}{l}q=r^{2} \\
q=r^{2}, r \equiv 3 \quad(\bmod 4)\end{array}$ & $\begin{array}{l}n \leq r \\
n=2 t r, t \leq(r-2) / 2\end{array}$ & 13 \\
\hline $\begin{array}{l}q \equiv 3 \quad(\bmod 4) \\
q \equiv 1 \quad(\bmod 4)\end{array}$ & $\begin{array}{l}n \equiv 3 \quad(\bmod 4),(n-1) \mid(q-1) \\
(n-1) \mid(q-1)\end{array}$ & 23 \\
\hline $\begin{array}{l}q \equiv 1 \quad(\bmod 4) \\
q \text { odd } \\
q \text { odd } \\
q=r^{2}, r \text { odd } \\
q=r^{2}, r \text { odd } \\
q=r^{s}, r \text { odd }, s \geq 2 \\
q=r^{s}, r \text { odd }, s \geq 2 \\
q=r^{s}, r \text { odd }, s \geq 2 \\
q=r^{s}, r \text { odd }, s \geq 2 \\
q=p^{m}, p \text { odd prime } \\
q=p^{m}, p \text { odd prime }\end{array}$ & $\begin{array}{l}n \mid(q-1), n<q-1 \\
(n-1) \mid(q-1), \eta(1-n)=1 \\
(n-2) \mid(q-1), \eta(2-n)=1 \\
n=t r, t \text { even }, 1 \leq t \leq r \\
n=t r+1, t \text { odd }, 1 \leq t \leq r \\
n=l r, l \text { even }, 2 l \mid(r-1) \\
n=l r, l \text { even },(l-1) \mid(r-1), \eta(1-l)=1 \\
n=l r+1, l \text { odd }, l \mid(r-1), \eta(l)=1 \\
n=l r+1, l \text { odd },(l-1) \mid(r-1), \eta(l-1)=\eta(-1)=1 \\
n=p r+1, r \mid m \\
n=2 p^{e}, 1 \leq e<m, \eta(-1)=1\end{array}$ & 24 \\
\hline $\begin{array}{l}q=p^{m} \\
q=p^{m}, m \text { even }, r=p^{s}, s \mid \frac{m}{2} \\
q=p^{m}, q \equiv 1 \quad(\bmod 4) \\
q=p^{m}, m \text { even }, r=p^{s}, s \mid \frac{m}{2} \\
q=p^{m}, q \equiv 1 \quad(\bmod 4) \\
q=p^{m}\end{array}$ & $\begin{array}{l}n \mid(q-1),(q-1) / n \text { even } \\
n=2 t r^{\ell}, 0 \leq \ell<m / s, 1 \leq t \leq(r-1) / 2 \\
n=2 p^{\ell}, 0 \leq \ell<m \\
n=(2 t+1) r^{\ell}+1,0 \leq \ell<m / s, 0 \leq t \leq(r-1) / 2 \text { or }(\ell, t)=(m / s, 0) \\
n=p^{\ell}+1,0 \leq \ell \leq m \\
(n-2) \mid(q-1), \eta(2-n)=1\end{array}$ & 7 \\
\hline $\begin{array}{l}q=p^{m} \\
q=p^{m}, q \equiv 1 \quad(\bmod 4) \\
q=p^{m}, q \equiv 1 \quad(\bmod 4) \\
q=p^{m}, q \text { a square } \\
q=p^{m}, q \text { a square } \\
q=p^{m}, q \text { a square } \\
q=p^{m} \\
q=p^{m}, q \equiv 1 \quad(\bmod 4) \\
q=p^{m}, q \equiv 1 \quad(\bmod 4) \\
q=p^{m}, m=2 m_{0} \\
q=p^{m}, m=2 m_{0} \\
q=p^{m}, q \text { a square } \\
q=p^{m}, q \text { a square } \\
q=p^{m}, q \text { a square }\end{array}$ & $\begin{array}{l}n=n_{0}, p\left|n_{0},\left(n_{0}-1\right)\right|(q-1) \\
n=n_{0}+1, p\left|n_{0},\left(n_{0}-1\right)\right|(q-1) \\
n=p^{r}+1,1 \leq r \leq m, r \mid m \\
n=n_{0},\left(n_{0}-1\right) \mid(q-1) \\
n=n_{0}+1,\left(n_{0}-1\right) \mid(q-1) \\
n=2 n_{0}, n_{0} \text { odd },\left(n_{0}-1\right) \mid(q-1) \\
n=n_{0}, n_{0} \mid \frac{(q-1)}{2} \\
n=n_{0}+1, n_{0} \mid \frac{(q-1)}{2} \\
n=2 p^{r}, 1 \leq r<m, r \mid m \\
n=(t+1) n_{0}+2, n_{0}=p^{m_{0}}-1, n_{0} \equiv 0 \quad(\bmod 4), t \text { odd }, 0<t \leq \frac{n_{0}}{2}+1 \\
n=(t+1) n_{0}+2, n_{0}=p^{m_{0}}-1, n_{0} \equiv 2 \quad(\bmod 4), 0<t \leq \frac{n_{0}}{2} \\
n=(t+1) n_{0}+2, n_{0}=\frac{q-1}{p^{r}+1} \text { even, } 1 \leq r<m, \frac{n_{0}\left(p^{r}+1\right)}{2\left(p^{r}-1\right)} \text { odd }, t \text { odd }, 1 \leq t \leq p^{r} \\
n=(t+1) n_{0}+2, n_{0}=\frac{q-1}{p^{r}+1} \text { even, } 1 \leq r<m, \frac{n_{0}\left(p^{r}+1\right)}{2\left(p^{r}-1\right)} \text { even }, 1 \leq t \leq p^{r} \\
n=(t+1) n_{0}+2, n_{0}=\frac{q-1}{p^{r}-1} \text { even, } 1 \leq r<m, r \mid \frac{m}{2}, 1 \leq t \leq p^{r}-2\end{array}$ & 19 \\
\hline
\end{tabular}


self-dual ones.

In this paper, we will discover more families of optimal self-dual codes from algbraic curves over finite fields. Optimal self-dual codes are constructed from rational points on the curves and embedding their orthogonal subcodes. We improve the construction [19] and other known constructions over $\mathbb{F}_{q}$ with $q$ a prime (see Theorem 21) and also give explict constructions of the cosets of $\mathbb{F}_{q}$ with desired properties (see Lemma 10 and Lemma 11). Due to Lemma 6] and Lemma 7, new classes of selfdual codes with prescribed minimum distance are constructed. Additionally, we construct MDS self-dual codes with new parameters $[24,12,13]_{37}$, $[32,16,17]_{41},[26,13,14]_{61},[42,21,22]_{61},[50,25,26]_{73},[24,12,13]_{81}$, almost MDS self-dual codes with new parameters $[16,8,8]_{9},[16,8,8]_{16},[18,9,9]_{16}$, $[20,10,10]_{16},[22,11,11]_{16},[24,12,12]_{16}$ and optimal self-dual codes with new parameters $[28,14,12]_{9},[26,13,12]_{16},[28,14,13]_{16},[30,15,14]_{16},[32,16,15]_{16}$.

The paper is organized as follows: Section 2 gives preliminaries and background on algebraic geometry codes. Section 3 provides explicit constructions of self-dual codes from various algebraic curves. New families of self-dual codes are presented as well some numerical examples are also given. We end up with concluding remark in Section 4 .

\section{Preliminaries}

Let $\mathbb{F}_{q}$ be the finite field with $q$ elements. A linear code of length $n$ and dimension $k$ over $\mathbb{F}_{q}$, denoted as $q$-ary $[n, k]$ code, is a $k$-dimensional subspace of $\mathbb{F}_{q}^{n}$. The (Hamming) weight $\mathrm{wt}(\mathbf{x})$ of a vector $\mathbf{x}=\left(x_{1}, \ldots, x_{n}\right)$ is the number of nonzero coordinates in it. The minimum distance (or minimum weight) $d(C)$ of $C$ is $d(C):=\min \{\operatorname{wt}(\mathbf{x}) \mid \mathbf{x} \in C, \mathbf{x} \neq \mathbf{0}\}$. The parameters of an $[n, k]$ code with minimum distance $d$ are written $[n, k, d]$. If $C$ is an $[n, k, d]$ code, then from the Singleton bound, its minimum distance is bounded above by

$$
d \leq n-k+1
$$

A code meeting the above bound is called Maximum Distance Separable (MDS). A code is called almost MDS if its minimum distance is one unit less than the MDS case. A code is called optimal if it has the highest possible minimum distance for its length and dimension. The Euclidean inner product of $\mathbf{x}=\left(x_{1}, \ldots, x_{n}\right)$ and $\mathbf{y}=\left(y_{1}, \ldots, y_{n}\right)$ in $\mathbb{F}_{q}^{n}$ is $\mathbf{x} \cdot \mathbf{y}=\sum_{i=1}^{n} x_{i} y_{i}$. The dual of $C$, denoted by $C^{\perp}$, is the set of vectors orthogonal to every 
codeword of $C$ under the Euclidean inner product. A linear code $C$ is called self-orthogonal if $C \subset C^{\perp}$ and self-dual if $C=C^{\perp}$. It is well-known that a self-dual code can only exist for even lengths.

We refer to Stichtenoth [21] for undefined terms related to algebraic function fields.

Let $\mathcal{X}$ be a smooth projective curve of genus $g$ over $\mathbb{F}_{q}$. The field of rational functions of $\mathcal{X}$ is denoted by $\mathbb{F}_{q}(\mathcal{X})$. Function fields of algebraic curves over a finite field can be characterized as finite separable extensions of $\mathbb{F}_{q}(x)$. We identify points on the curve $\mathcal{X}$ with places of the function field $\mathbb{F}_{q}(\mathcal{X})$. A point on $\mathcal{X}$ is called rational if all of its coordinates belong to $\mathbb{F}_{q}$. Rational points can be identified with places of degree one. We denote the set of $\mathbb{F}_{q}$-rational points of $\mathcal{X}$ by $\mathcal{X}\left(\mathbb{F}_{q}\right)$.

A divisor $G$ on the curve $\mathcal{X}$ is a formal sum $\sum_{P \in \mathcal{X}} n_{P} P$ with only finitely many nonzeros $n_{P} \in \mathbb{Z}$. The support of $G$ is defined as $\operatorname{supp}(G):=\left\{P \mid n_{P} \neq\right.$ $0\}$. The degree of $G$ is defined by $\operatorname{deg}(G):=\sum_{P \in \mathcal{X}} n_{P} \operatorname{deg}(P)$. For two divisors $G=\sum_{P \in \mathcal{X}} n_{P} P$ and $H=\sum_{P \in \mathcal{X}} m_{P} P$, we say that $G \geq H$ if $n_{P} \geq m_{P}$ for all places $P \in \mathcal{X}$.

It is well-known that a nonzero polynomial $f(x) \in \mathbb{F}_{q}(x)$ can be factorized into irreducible factors as $f(x)=\alpha \prod_{i=1}^{s} p_{i}(x)^{e_{i}}$, with $\alpha \in \mathbb{F}_{q}^{*}$. Moreover, any irreducible polynomial $p_{i}(x)$ corresponds to a place, say $P_{i}$. We define the valuation of $f$ at $P_{i}$ as $v_{P_{i}}(f):=t$ if $p_{i}(x)^{t} \mid f(x)$ but $p_{i}(x)^{(t+1)} \not f f(x)$.

For a nonzero rational function $f$ on the curve $\mathcal{X}$, we define the "principal" divisor of $f$ as

$$
(f):=\sum_{P \in \mathcal{X}} v_{P}(f) P .
$$

If $Z(f)$ and $N(f)$ denotes the set of zeros and poles of $f$ respectively, we define the zero divisor and pole divisor of $f$, respectively by

$$
\begin{aligned}
(f)_{0} & :=\sum_{P \in Z(f)} v_{P}(f) P, \\
(f)_{\infty} & :=\sum_{P \in N(f)}-v_{P}(f) P .
\end{aligned}
$$

Then $(f)=(f)_{0}-(f)_{\infty}$, and it is well-known that the principal divisor $f$ has degree 0 .

We say that two divisors $G$ and $H$ on the curve $\mathcal{X}$ are equivalent if $G=H+(z)$ for some rational function $z \in \mathbb{F}_{q}(\mathcal{X})$. 
For a divisor $G$ on the curve $\mathcal{X}$, we define

$$
\mathcal{L}(G):=\left\{f \in \mathbb{F}_{q}(\mathcal{X}) \backslash\{0\} \mid(f)+G \geq 0\right\} \cup\{0\}
$$

and

$$
\Omega(G):=\{\omega \in \Omega \backslash\{0\} \mid(\omega)-G \geq 0\} \cup\{0\},
$$

where $\Omega:=\left\{f d x \mid f \in \mathbb{F}_{q}(\mathcal{X})\right\}$, the set of differential forms on $\mathcal{X}$. It is wellknown that, for a differential form $\omega$ on $\mathcal{X}$, there exists a unique a rational function $f$ on $\mathcal{X}$ such that

$$
\omega=f d t
$$

where $t$ is a local uniformizing parameters. In this case, we define the divisor associated to $\omega$ by

$$
(\omega)=\sum_{P \in \mathcal{X}} v_{P}(\omega) P
$$

where $v_{P}(\omega):=v_{P}(f)$.

Through out the paper, we let $D=P_{1}+\cdots+P_{n}$, called the rational divisor, where $\left(P_{i}\right)_{1 \leq i \leq n}$ are places of degree one, and $G$ a divisor with $\operatorname{supp}(D) \cap \operatorname{supp}(G)=\emptyset$. Define the algebraic geometry code by

$$
C_{\mathcal{L}}(D, G):=\left\{\left(f\left(P_{1}\right), \ldots, f\left(P_{n}\right)\right) \mid f \in \mathcal{L}(G)\right\},
$$

and the differential algebraic geometry code as

$$
C_{\Omega}(D, G):=\left\{\left(\operatorname{Res}_{P_{1}}(\omega), \ldots, \operatorname{Res}_{P_{n}}(\omega)\right) \mid \omega \in \Omega(G-D)\right\},
$$

where $\operatorname{Res}_{P}(\omega)$ denotes the residue of $\omega$ at point $P$.

The parameters of an algebraic geometry code $C_{\mathcal{L}}(D, G)$ is given as follows.

Theorem 1. [21, Corollary 2.2.3] Assume that $2 g-2<\operatorname{deg}(G)<n$. Then the code $C_{\mathcal{L}}(D, G)$ has parameters $[n, k, d]$ satisfying

$$
k=\operatorname{deg}(G)-g+1 \text { and } d \geq n-\operatorname{deg}(G) .
$$

The dual of the algebraic geometry code $C_{\mathcal{L}}(D, G)$ can be described as follows.

Lemma 1. [21, Theorem 2.2.8] With above notation, the two codes $C_{\mathcal{L}}(D, G)$ and $C_{\Omega}(D, G)$ are dual to each other. 
Moreover, the differential code $C_{\Omega}(D, G)$ is determined as follows.

Lemma 2. [21, Proposition 2.2.10] With the above notation, assume that there exists a differential form $\omega$ satisfying

1. $v_{P_{i}}(\omega)=-1,1 \leq i \leq n$ and

2. $\operatorname{Res}_{P_{i}}(\omega)=\operatorname{Res}_{P_{j}}(\omega)$ for $1 \leq i \leq n$.

Then $C_{\Omega}(D, G)=a \cdot C_{\mathcal{L}}(D, D-G+(\omega))$ for some $a \in\left(\mathbb{F}_{q}^{*}\right)^{n}$.

\section{Self-dual algebraic geometry codes}

In this section, we will construct self-dual codes from algebraic geometry codes. Self-dual codes can be constructed directly from Lemma 3 or from their self-orthogonal subcodes by extending the basis of the existing codes.

Lemma 3. [20, Corollary 3.4] With the above notation, assume that there exists a differential form $\omega$ satisfying

1. $v_{P_{i}}(\omega)=-1,1 \leq i \leq n$ and

2. $\operatorname{Res}_{P_{i}}(\omega)=\operatorname{Res}_{P_{j}}(\omega)=a_{i}^{2}, 1 \leq i \leq n$, for some $a_{i} \in \mathbb{F}_{q}^{*}$.

Then the following statements hold.

1. If $2 G \leq D+(\omega)$, then there exists a divisor $G^{\prime}$ such that $C_{\mathcal{L}}(D, G) \sim$ $C_{\mathcal{L}}\left(D, G^{\prime}\right)$, and $C_{\mathcal{L}}\left(D, G^{\prime}\right)$ is self-orthogonal.

2. If $2 G=D+(\omega)$, then there exists a divisor $G^{\prime}$ such that $C_{\mathcal{L}}(D, G) \sim$ $C_{\mathcal{L}}\left(D, G^{\prime}\right)$, and $C_{\mathcal{L}}\left(D, G^{\prime}\right)$ is self-dual.

The existence of self-dual algebraic geometry codes can be given as follows.

Proposition 1. [22, Corollary 3.1.49, p.292] With the above notation, assume that $N=\left|\mathcal{X}\left(\mathbb{F}_{q}\right)\right|>2 g$. Then there exists a self-dual code with parameters $\left[n, \frac{n}{2}, \geq \frac{n}{2}-g+1\right]$ over $\mathbb{F}_{q}$ for some $n$ even such that $n \geq N-2 g-1$.

The following lemma will be applied many times for constructing a $q$-ary self-dual code of length $n$ (if it exists for such a length). 
Lemma 4. Let $n$ be an odd positive integer and $C$ a q-ary self-orthogonal code with parameters $\left[n, \frac{n-1}{2}\right]$. Then there exists a self-orthogonal code $C_{0}$ with parameters $\left[n+1, \frac{n-1}{2}\right]$ and a self-dual code $C_{0}^{\prime}$ with parameters $\left[n+1, \frac{n+1}{2}\right]$ such that $C_{0} \subset C_{0}^{\prime} \subset C_{0}^{\perp}$.

Proof. Let $G$ be the generator matrix of $C$ and $C_{0}$ be a self-orthogonal code obtained from $C$ by lengthening one zero coordinate. Clearly, the code $C_{0}$ has parameters $\left[n+1, \frac{n-1}{2}\right]$, and $C_{0}^{\perp}$ has parameters $\left[n+1, \frac{n+1}{2}+1\right]$. Denote $G_{0}$ the generator matrix of $C_{0}$, that is,

$$
G_{0}=\left(\begin{array}{cc} 
& 0 \\
G & \vdots \\
& 0
\end{array}\right)
$$

Let $\mathbf{x}$ be a nonzero element in the quotient space $C_{0}^{\perp} / C_{0}$ such that $\mathbf{x} \cdot \mathbf{x}=0$. Then the code $C_{0}^{\prime}$ with its following generator matrix $G_{0}^{\prime}$ is self-dual with parameters $\left[n+1, \frac{n+1}{2}\right]$ :

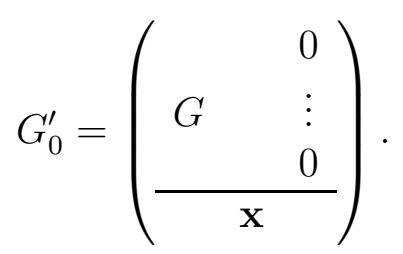

Moreover, we have the following inclusion

$$
C_{0} \subset C_{0}^{\prime} \subset C_{0}^{\perp}
$$

Similarly, we have the following embedding.

Lemma 5. Let $n$ be an even positive integer and $C$ a q-ary self-orthogonal code with parameters $\left[n, \frac{n}{2}-1\right]$. Then there exists a self-dual code $C^{\prime}$ (if it exists for such a length) with parameters $\left[n, \frac{n}{2}\right]$ such that $C \subset C^{\prime} \subset C^{\perp}$.

Lemma 6. Let $\mathcal{X}$ be a smooth projective curve having genus $g$. Let $n$ be an odd positive integer and $D=P_{1}+\cdots+P_{n}$ be a divisor on $\mathcal{X}$. Assume that there exists a differential form $\omega$ satisfying

1. $v_{P_{i}}(\omega)=-1$, for $i=1, \ldots, n$ and 
2. $\left.\operatorname{Res}_{P_{i}}(\omega)=\operatorname{Res}_{P_{j}}(\omega)\right)=a_{i}^{2}$ with $a_{i} \in \mathbb{F}_{q}^{*}$ for $1 \leq i, j \leq n$.

If $G=\frac{(2 g-3+n)}{2} P_{\infty}$ with $\operatorname{supp}(G) \cap \operatorname{supp}(D)=\emptyset$, then there exists a selforthogonal code $C_{\mathcal{L}}(D, G)$ with parameters $\left[n, \frac{n-1}{2}, \frac{n+3}{2}-g\right]$. Moreover, the code $C_{\mathcal{L}}(D, G)$ can be embedded into a self-dual $\left[n+1, \frac{n+1}{2}, \geq \frac{n+1}{2}-g\right]$ code $C^{\prime}$ (if a self-dual code exists for such a length $n$ ).

Proof. Choose $U$ as a subset of $\mathbb{F}_{q}$ with its size $|U|=n$ so that $\omega=\frac{d x}{h}$, where $h(x)=\prod_{\alpha \in U}(x-\alpha)$, satisfying the above two conditions. Then the divisor $(\omega)=(2 g-2+n) P_{\infty}-D$, and thus $2 G \leq(\omega)+D$. From Lemma 3 and Theorem 1, there exists a self-orthogonal code $C_{\mathcal{L}}(D, G)$ with parameters $\left[n, \frac{n-1}{2}, \geq \frac{n+3}{2}-g\right]$. The second assertion follows from Lemma 4. First note that

$$
\begin{aligned}
C_{\mathcal{L}}(D, G)^{\perp} & =a \cdot C_{\mathcal{L}}(D, D-G+(\omega))(\text { from Lemma 2) } \\
& =a \cdot C_{\mathcal{L}}\left(D, \frac{(2 g-1+n)}{2} P_{\infty}\right)
\end{aligned}
$$

We now calculate the lower bound on the minimum distance of the dual code.

$$
\begin{aligned}
d\left(C_{\mathcal{L}}(D, G)^{\perp}\right) & \geq n-\frac{(2 g-1+n)}{2}(\text { due to (2) and Theorem 1) } \\
& =\frac{n+1}{2}-g .
\end{aligned}
$$

The minumum distance of $C^{\prime}$ follows from the fact that $C^{\prime} \subset C_{\mathcal{L}}^{\perp}(D, G)$, and this completes the proof.

Lemma 7. Let $\mathcal{X}$ be a smooth projective curve having genus $g$. Let $n$ be an even positive integer and $D=P_{1}+\cdots+P_{n}$ be a divisor on $\mathcal{X}$. Assume that there exists a differential form $\omega$ satisfying

1. $v_{P_{i}}(\omega)=-1$, for $i=1, \ldots, n$ and

2. $\left.\operatorname{Res}_{P_{i}}(\omega)=\operatorname{Res}_{P_{j}}(\omega)\right)=a_{i}^{2}$ with $a_{i} \in \mathbb{F}_{q}^{*}$ for $1 \leq i, j \leq n$.

If $G=\frac{(2 g-2+n)}{2} P_{\infty}$ with $\operatorname{supp}(G) \cap \operatorname{supp}(D)=\emptyset$, then there exists a selforthogonal code $C_{\mathcal{L}}(D, G)$ with parameters $\left[n, \frac{n}{2}, \frac{n}{2}+1-g\right]$.

Proof. The result follows from the same reasoning as that in Lemma 6 . 


\subsection{Self-dual codes from projective lines}

In this subsection, we will discover new families of MDS self-dual codes based on the work from [19]. In what follows, we let for $a \in \mathbb{F}_{q}, \eta(a):=1$ if $a$ is a square in $\mathbb{F}_{q}$, and $\eta(a):=-1$ if $a$ is not a square in $\mathbb{F}_{q}$.

The following two lemmas [19] will be used to construct self-dual codes of genus zero.

Lemma 8. [19, Lemma 6] For $G=s P_{\infty}$ with $s \leq\left\lfloor\frac{n-2}{2}\right\rfloor$, if $\left(h^{\prime}\left(P_{i}\right)\right)_{1 \leq i \leq n}$ are squares in $\mathbb{F}_{q}^{*}$, then $C_{\mathcal{L}}\left(D, G-\left(1 / \sqrt{h^{\prime}}\right)\right)$ is an $M D S$ self-orthogonal code.

The following lemma is useful for constructing a self-dual code from its self-orthogonal subcode.

Lemma 9. [19, Lemma 7] Let $q \equiv 1(\bmod 4)$. Assume that $G=(k-$ 1) $P_{\infty}, n=2 k+1$, and $\left(h^{\prime}\left(P_{i}\right)\right)_{1 \leq i \leq n}$ are squares in $\mathbb{F}_{q}^{*}$. Then the $q$-ary selforthogonal code $C_{\mathcal{L}}\left(D, G-\left(1 / \sqrt{h^{\prime}}\right)\right)$ with parameters $[n, k]$ can be embedded into a q-ary $M D S$ self-dual $[n+1, k+1]$ code.

Now, we construct MDS self-dual codes from Lemma 8 and Lemma 9 .

Theorem 2. Let $q=p^{m}$ be an odd prime power. If $\eta(-1)=\eta(n)=1$, $n \mid(q-1)$ and $n$ even, then there exists an $[2 n+2, n+1, n+2]$ self-dual code over $\mathbb{F}_{q}$.

Proof. Let $U_{n}=\left\{\alpha \in \mathbb{F}_{q}^{*} \mid \alpha^{n}=1\right\}$. Let $\beta_{1} \in \mathbb{F}_{q}^{*}$ such that $\beta_{1}^{n}-1$ is a nonzero square in $\mathbb{F}_{q}$. Put $U=U_{n} \cup \beta_{1} U_{n} \cup\{0\}$, and write

$$
h(x)=\prod_{\beta \in U}(x-\beta) .
$$

Then we have that

$$
h^{\prime}(x)=\left((n+1) x^{n}-1\right)\left(x^{n}-\beta_{1}^{n}\right)+n x^{n}\left(x^{n}-1\right) .
$$

Consider the following quadratic equation

$$
a^{2}+b^{2}=1
$$

For any $q$, (3) has $T=(q-1)-4$ solutions, say $\left(a_{1}, \pm b_{1}\right), \ldots,\left(a_{\frac{T}{2}}, \pm b_{\frac{T}{2}}\right)$, with $\left(a_{i}, b_{i}\right) \neq(0, \pm 1),( \pm 1,0)$. Take $\beta_{1}=\sqrt[n]{a_{i}^{2}}$ for some $1 \leq i \leq t,\left(t<\frac{T}{2}\right)$. Then we get $1-\beta_{1}^{n}=1-a_{i}^{2}=b_{i}^{2}$ which are squares in $\mathbb{F}_{q}^{*}$. 
We have that $-1, n$ are squares in $\mathbb{F}_{q}$. Moreover, since $\beta_{1}^{n}$ and $\left(\beta_{1}^{n}-1\right)$ are squares in $\mathbb{F}_{q}^{*}$, it implies that $h^{\prime}(\beta)$ is a square in $\mathbb{F}_{q}^{*}$ for any $\beta \in U$. Now, the fact that all the roots of $h(x)$ are simple gives rise to a self-orthogonal code with parameters $[2 n+1, n, n+2]$. Thus, by Lemma 9, it can be embedded into a $q$-ary self-dual code with parameters $[2 n+2, n+1, n+2]$.

Example 1. We construct MDS self-dual codes with new parameters as follows.

1. Taking $q=37, n=12$, we obtain a self-dual code over $\mathbb{F}_{37}$ with parameters $[26,13,14]$.

2. Taking $q=61, n=12,20$ we obtain self-dual code over $\mathbb{F}_{61}$ with parameters $[26,13,14],[42,21,22]$, respectively.

3. Taking $q=73, n=24$, we obtain a self-dual code over $\mathbb{F}_{73}$ with parameters $[50,25,26]$.

Remark 1. In the proof of Theorem 2, we have found many values of $\beta_{i}$ such that $1-\beta_{i}^{n}$ is a square. Furthermore, if there exist $\beta_{1}$ and $\beta_{2}$ such that $\beta_{1}^{n}-\beta_{2}^{n}$ is again a square, then we can construct an MDS self-dual code of length $3 n+2$ over $\mathbb{F}_{q}$. For example, taking $q=41, n=10$ and considering two non-zero multiplicative cosets of $U_{n}$ yields a self-dual code over $\mathbb{F}_{41}$ with parameters $[32,16,17]$. The generator matrix of the self-dual code over $\mathbb{F}_{41}$ is given as follows.

$$
\left(\begin{array}{ccccccccccccccccc} 
& 20 & 15 & 20 & 11 & 6 & 11 & 22 & 10 & 39 & 15 & 5 & 9 & 20 & 31 & 33 & 40 \\
37 & 5 & 11 & 12 & 3 & 15 & 4 & 10 & 37 & 18 & 35 & 8 & 3 & 26 & 32 & 8 \\
12 & 29 & 9 & 14 & 13 & 11 & 8 & 30 & 16 & 20 & 17 & 22 & 3 & 9 & 13 & 10 \\
2 & 17 & 11 & 25 & 14 & 3 & 1 & 27 & 38 & 5 & 1 & 15 & 36 & 2 & 1 & 21 \\
27 & 21 & 21 & 13 & 20 & 7 & 36 & 15 & 29 & 30 & 25 & 20 & 1 & 11 & 2 & 32 \\
39 & 7 & 2 & 1 & 26 & 25 & 5 & 38 & 38 & 13 & 33 & 20 & 17 & 15 & 7 & 36 \\
I_{11} & 40 & 39 & 34 & 15 & 18 & 12 & 6 & 28 & 25 & 10 & 21 & 23 & 8 & 35 & 26 & 26 \\
& 30 & 36 & 28 & 2 & 1 & 11 & 12 & 28 & 2 & 27 & 34 & 35 & 4 & 4 & 20 & 2 \\
& 22 & 18 & 5 & 24 & 5 & 40 & 23 & 9 & 34 & 40 & 12 & 34 & 9 & 34 & 33 & 31 \\
20 & 13 & 9 & 12 & 31 & 35 & 37 & 33 & 26 & 37 & 23 & 39 & 29 & 18 & 25 & 19 \\
& 11 & 19 & 18 & 16 & 38 & 40 & 2 & 29 & 8 & 30 & 30 & 10 & 12 & 2 & 20 & 30 \\
34 & 13 & 10 & 13 & 18 & 28 & 19 & 14 & 28 & 31 & 4 & 34 & 24 & 9 & 31 & 35 \\
24 & 31 & 21 & 40 & 12 & 23 & 25 & 4 & 17 & 27 & 13 & 4 & 31 & 40 & 23 & 30 \\
& 40 & 31 & 36 & 35 & 28 & 38 & 21 & 31 & 14 & 20 & 16 & 36 & 20 & 37 & 34 & 21 \\
9 & 10 & 23 & 11 & 36 & 23 & 30 & 9 & 16 & 22 & 27 & 32 & 37 & 26 & 39 & 26 \\
36 & 4 & 32 & 32 & 4 & 4 & 10 & 14 & 12 & 14 & 20 & 30 & 29 & 34 & 8 & 21
\end{array}\right) .
$$

The two following lemmas play the key role in determining whether the difference of two special elements in $\mathbb{F}_{q}$ is a square or not and also in determining the number of cosets of a multiplicate subgroup of $\mathbb{F}_{q}^{*}$. 
Lemma 10. Let $q=p^{m}$ with $p$ an odd prime, $n=\frac{q-1}{p^{r}+1}$ and for $\alpha_{i}, \alpha_{j} \in \mathbb{F}_{q}$ with $\alpha_{i} \neq \alpha_{j}$, denote $\alpha_{i j}=\alpha_{i}^{n}-\alpha_{j}^{n}$. Then for $\omega$ a primitive element of $\mathbb{F}_{q}$, we have the following equality:

$$
\alpha_{i j}=\frac{\omega^{\frac{n\left(p^{r}+1\right)}{2\left(p^{r}-1\right)}}}{\alpha_{i} \alpha_{j}} .
$$

Proof. Raising $\alpha_{i j}$ to the power $p^{r}-1$, we get

$$
\begin{aligned}
\alpha_{i j}^{p^{r}-1} & =\frac{\left(\alpha_{i}^{n}-\alpha_{j}^{n}\right)^{p^{r}}}{\alpha_{i}^{n}-\alpha_{j}^{n}}=\frac{\left(\alpha_{i}^{n p^{r}}-\alpha_{j}^{n} p^{r}\right)}{\alpha_{i}^{n}-\alpha_{j}^{n}} \\
& =\frac{\left(\alpha_{i}^{q-1-n}-\alpha_{j}^{q-1-n}\right)}{\alpha_{i}^{n}-\alpha_{j}^{n}}=\frac{\frac{1}{\alpha_{i}^{n}}-\frac{1}{\alpha_{j}^{n}}}{\alpha_{i}^{n}-\alpha_{j}^{n}} \\
& =\frac{\omega \frac{n\left(p^{r}+1\right)}{\alpha_{i}^{n} \alpha_{j}^{n}}}{\alpha_{i}^{n}}
\end{aligned}
$$

where the last equality come from the fact that $\omega^{\frac{q-1}{2}}=-1$.

By taking the $\left(p^{r}-1\right)$-th root, the result follows.

Lemma 11. Let $q=p^{m}$ with $p$ an odd prime, $r \mid m, n=\frac{q-1}{p^{r}-1}$ and for $\alpha_{i}, \alpha_{j} \in$ $\mathbb{F}_{q}$ with $\alpha_{i} \neq \alpha_{j}$, denote $\alpha_{i j}=\alpha_{i}^{n}-\alpha_{j}^{n}$. Then for $\omega$ a primitive element of $\mathbb{F}_{q}$, we have the following:

$$
\alpha_{i j} \in \mathbb{F}_{p^{r}} .
$$

Proof. Raising $\alpha_{i j}$ to the power $p^{r}$, we get

$$
\alpha_{i j}^{p^{r}}=\left(\alpha_{i}^{n p^{r}}-\alpha_{j}^{n p^{r}}\right)=\left(\alpha_{i}^{q-1+n}-\alpha_{j}^{q-1+n}\right)=\alpha_{i}^{n}-\alpha_{j}^{n}=\alpha_{i j} .
$$

Thus the result follows.

Theorem 3. Let $q=p^{m}$ be an odd square and $n$ even. Put $s=(t+1) n$.

1. If $\frac{n\left(p^{r}+1\right)}{2\left(p^{r}-1\right)}$ is even, then there exists a self-dual code over $\mathbb{F}_{q}$ with parameters $\left[s, \frac{s}{2}, \frac{s}{2}+1\right]$, with $n=\frac{q-1}{p^{r}+1}$, for $1 \leq t \leq p^{r}$.

2. If $\frac{n\left(p^{r}+1\right)}{2\left(p^{r}-1\right)}$ is odd, then there exists a self-dual code over $\mathbb{F}_{q}$ with parameters $\left[s, \frac{s}{2}, \frac{s}{2}+1\right]$, with $n=\frac{q-1}{p^{r}+1}$, for $t$ odd and $1 \leq t \leq p^{r}$.

3. There exists a self-dual code over $\mathbb{F}_{q}$ with parameters $\left[s, \frac{s}{2}, \frac{s}{2}+1\right]$, with $n=\frac{q-1}{p^{r}-1}, r \mid \frac{m}{2}$, for $1 \leq t \leq p^{r}-2$. 
Proof. Let $U_{n}$ be a multiplicative subgroup of $\mathbb{F}_{q}^{*}$ of order $n$, say $U_{n}=$ $\left\{u_{1}, \ldots, u_{n}\right\}$. Let $\alpha_{1} U_{n}, \ldots, \alpha_{t} U_{n}$ be $t$ nonzero cosets of $U_{n}$, where $\left(\alpha_{i}\right)_{1 \leq i \leq t}$ will be determined later. Put $U=U_{n} \cup\left(\bigcup_{i=1}^{t} \alpha_{i} U_{n}\right)$, and write

$$
h(x)=\prod_{\alpha \in U}(x-\alpha) .
$$

Clearly, all the roots of $h(x)$ are simple. The derivative of $h(x)$ is given by

$$
h^{\prime}(x)=n x^{n-1} \prod_{i=1}^{t}\left(x^{n}-\alpha_{i}^{n}\right)+n x^{n-1}\left(x^{n}-1\right)\left(\sum_{i=1}^{t} \prod_{j=1, j \neq i}^{t}\left(x^{n}-\alpha_{j}^{n}\right)\right) .
$$

For $1 \leq j \leq t, 1 \leq s \leq n$, we have

$$
\begin{aligned}
& h^{\prime}\left(u_{s}\right) \quad=n u_{s}^{n-1}\left(\alpha_{j}^{n}-1\right) \prod_{i=1, i \neq j}^{t}\left(1-\alpha_{i}^{n}\right), \\
& h^{\prime}\left(\alpha_{j} u_{s}\right)=n\left(\alpha_{j} u_{s}\right)^{n-1}\left(\alpha_{j}^{n}-1\right) \prod_{i=1, i \neq j}^{t}\left(\alpha_{j}^{n}-\alpha_{i}^{n}\right) .
\end{aligned}
$$

For $1 \leq i, j \leq t$ and $n=\frac{q-1}{p^{r}+1}$, we have from (44)

$$
\alpha_{i j}=\alpha_{i}^{n}-\alpha_{j}^{n}=\frac{\omega^{\frac{n\left(p^{r}+1\right)}{2\left(p^{r}-1\right)}}}{\alpha_{i} \alpha_{j}},
$$

where $\omega$ is a primitive element of $\mathbb{F}_{q}$.

Fixing $j$ and taking all the product of $\alpha_{i j}$ for $1 \leq i \leq t, i \neq j$, we get that

$$
\prod_{i=1, i \neq j}^{t}\left(\alpha_{j}^{n}-\alpha_{i}^{n}\right)=\prod_{i=1, i \neq j}^{n} \frac{\omega^{\frac{n\left(p^{r}+1\right)}{2\left(p^{r}-1\right)}}}{\alpha_{i} \alpha_{j}} .
$$

Obviously, $n$ and $\left(u_{s}\right)_{1 \leq s \leq n}$ are squares in $\mathbb{F}_{q}$ for $q$ a square. Now, the squareness of $h^{\prime}\left(u_{s}\right)$ and $h^{\prime}\left(\alpha_{j} u_{s}\right)$ depend on the parity of $T=\frac{n\left(p^{r}+1\right)}{2\left(p^{r}-1\right)}$.

If $T$ is even, then $\alpha_{i}$ is chosen to be a square element in $\mathbb{F}_{q}$, and thus $\left(1-\alpha_{i}^{n}\right)$ and $\left(\alpha_{j}^{n}-\alpha_{i}^{n}\right)$ are square elements in $\mathbb{F}_{q}$ due to (44) of Lemma 10.

If $T$ is odd, then $\alpha_{i}$ is chosen to be a non-square element in $\mathbb{F}_{q}$, and thus $\left(1-\alpha_{i}^{n}\right)$ and $\left(\alpha_{j}^{n}-\alpha_{i}^{n}\right)$ are again square elements in $\mathbb{F}_{q}$ due to (4).

In conclusion, we have 
1. If $\frac{n\left(p^{r}+1\right)}{2\left(p^{r}-1\right)}$ is even, then $h^{\prime}\left(u_{s}\right)$ and $h^{\prime}\left(\alpha_{j} u_{s}\right)$ are squares in $\mathbb{F}_{q}^{*}$ for $1 \leq$ $s \leq n$ and $1 \leq j \leq t$ with $t \in\left\{1, \ldots, p^{r}\right\}$.

2. If $\frac{n\left(p^{r}+1\right)}{2\left(p^{r}-1\right)}$ is odd, then $h^{\prime}\left(u_{s}\right)$ and $h^{\prime}\left(\alpha_{j} u_{s}\right)$ are squares in $\mathbb{F}_{q}^{*}$ for $1 \leq s \leq n$ and $1 \leq j \leq t$ with $t$ odd and $t \in\left\{1, \ldots, p^{r}\right\}$.

For $1 \leq i, j \leq t$ and $n=\frac{q-1}{p^{r}-1}$, from (15) in Lemma 11, we get that $\alpha_{i j}=$ $\alpha_{i}^{n}-\alpha_{j}^{n} \in \mathbb{F}_{p^{r}}$, and hence it is a square if $r \mid \frac{m}{2}$. We have shown that $h^{\prime}(\alpha)$ is a nonzero square in $\mathbb{F}_{q}$ for any $\alpha \in U$, and thus the constructed code is self-dual by Lemma 8 .

Example 2. Taking $q=9^{2}, n=\frac{9^{2}-1}{9+1}=8, t=2$, we get an MDS self-dual with parameters $[24,12,13]$. These parameters are new. The generator matrix of the code is given as follows.

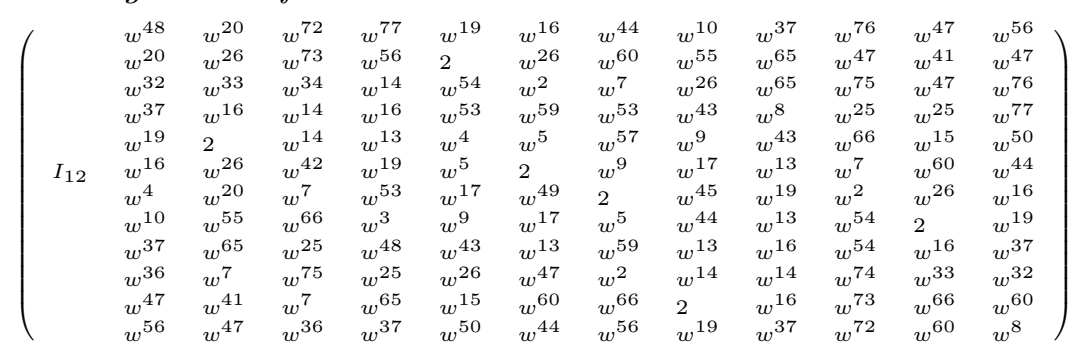

Theorem 4. Let $q$ be an odd prime power with $q \equiv 1(\bmod 4), 1 \leq r<$ $m, r \mid \frac{m}{2}$ and $n$ even with $\eta(n)=1$. Put $s=(t+1) n$.

1. If $\frac{n\left(p^{r}+1\right)}{2\left(p^{r}-1\right)}$ is odd, then there exists a self-dual code over $\mathbb{F}_{q}$ with parameters $\left[s, \frac{s}{2}, \frac{s}{2}+1\right]$, with $n=\frac{q-1}{p^{r}+1}$, for $t$ odd and $1 \leq t \leq p^{r}$.

2. If $\frac{n\left(p^{r}+1\right)}{2\left(p^{r}-1\right)}$ is even, then there exists a self-dual code over $\mathbb{F}_{q}$ with parameters $\left[s, \frac{s}{2}, \frac{s}{2}+1\right]$, with $n=\frac{q-1}{p^{r}+1}$, for $1 \leq t \leq p^{r}$.

3. There exists a self-dual code over $\mathbb{F}_{q}$ with parameters $\left[s, \frac{s}{2}, \frac{s}{2}+1\right]$, with $n=\frac{q-1}{p^{r}-1}, r \mid \frac{m}{2}$, for $1 \leq t \leq p^{r}-2$.

Proof. The proof follows from that of Theorem 3 , 
Table 2: Numbers of rational points of elliptic curves

\begin{tabular}{c|c|c} 
Elliptic curve $\mathcal{E}_{1, b, c}$ & $m$ & $\# \mathcal{E}_{1, b, c}\left(\mathbb{F}_{2^{m}}\right)$ \\
\hline \multirow{2}{*}{$y^{2}+y=x^{3}$} & $m \operatorname{odd}$ & $q+1-2 \sqrt{q}$ \\
& $m \equiv 0(\bmod 4)$ & $q+1-2 \sqrt{q}$ \\
& $m \equiv 2(\bmod 4)$ & $q+1+2 \sqrt{q}$ \\
\hline$y^{2}+y=x^{3}+x$ & $m \equiv 1,7(\bmod 8)$ & $q+1+2 \sqrt{q}$ \\
& $m \equiv 3,5(\bmod 8)$ & $q+1-2 \sqrt{q}$ \\
\hline$y^{2}+y=x^{3}+x+1$ & $m \equiv 1,7(\bmod 8)$ & $q+1+2 \sqrt{q}$ \\
& $m \equiv 3,5(\bmod 8)$ & $q+1-2 \sqrt{q}$ \\
\hline$y^{2}+y=x^{3}+b x\left(\operatorname{Tr}_{1}^{m}(b)=1\right)$ & $m \operatorname{even}$ & $q+1$ \\
\hline$y^{2}+y=x^{3}+c\left(\operatorname{Tr}_{1}^{m}(c)=1\right)$ & $m \equiv 0(\bmod 4)$ & $q+1+2 \sqrt{q}$ \\
& $m \equiv 2(\bmod 4)$ & $q+1-2 \sqrt{q}$
\end{tabular}

\subsection{Self-dual codes from elliptic curves and hyper-elliptic curves}

In this subsection, we will consider elliptic curves and hyper-elliptic curves over $\mathbb{F}_{q}, q$ even.

First, we will consider elliptic curves in Weierstrass form to construct self-dual codes. Let $q=p^{m}$ and an elliptic curve defined by the equation

$$
\mathcal{E}_{a, b, c}: y^{2}+a y=x^{3}+b x+c,
$$

where $a, b, c \in \mathbb{F}_{q}$. Let $S$ be the set of $x$-components of the affine points of $\mathcal{E}_{a, b, c}$ over $\mathbb{F}_{q}$, that is,

$$
S_{a, b, c}:=\left\{\alpha \in \mathbb{F}_{q} \mid \exists \beta \in \mathbb{F}_{q} \text { such that } \beta^{2}+a \beta=\alpha^{2}+b \alpha+c\right\} .
$$

For $q=2^{m}$, any $\alpha \in S_{1, b, c}$ gives exactly two points with $x$-component $\alpha$, and we denote these two points corresponding to $\alpha$ by $P_{\alpha}^{(1)}$ and $P_{\alpha}^{(2)}$. Then the set of all rational points of $\mathcal{E}_{1, b, c}$ over $\mathbb{F}_{q}$ is $\left\{P_{\alpha}^{(1)} \mid \alpha \in S_{1, b, c}\right\} \cup\left\{P_{\alpha}^{(2)} \mid \alpha \in\right.$ $\left.S_{1, b, c}\right\} \cup\left\{P_{\infty}\right\}$. The numbers of rational points of elliptic curves $\mathcal{E}$ over $\mathbb{F}_{q}$ are given in Table 2 .

Lemma 12 (Hilbert's Theorem 90). Let $q=p^{m}$. The equation $y^{p}-y=k$ has solutions over $\mathbb{F}_{q}$ if and only if $\operatorname{Tr}_{\mathbb{F}_{q} / \mathbb{F}_{p}}(k)=0$. 
Lemma 13. Let $q_{0}=2^{m}, m \geq 2$ and $q=q_{0}^{2}$. If $\alpha$ is an element in $\mathbb{F}_{q_{0}}$, then $\operatorname{Tr}_{\mathbb{F}_{q} / \mathbb{F}_{2}}(\alpha)=0$, and $\operatorname{Tr}_{\mathbb{F}_{q} / \mathbb{F}_{2}}\left(\alpha+\alpha^{3}\right)=0$.

Proof. For any $\alpha \in \mathbb{F}_{q_{0}}$, we have

$$
\begin{aligned}
\operatorname{Tr}_{\mathbb{F}_{q} / \mathbb{F}_{2}}(\alpha) & =\operatorname{Tr}_{\mathbb{F}_{q_{0}} / \mathbb{F}_{2}}\left(\operatorname{Tr}_{\mathbb{F}_{q} / \mathbb{F}_{q_{0}}}(\alpha)\right) \\
& =\operatorname{Tr}_{\mathbb{F}_{q_{0}} / \mathbb{F}_{2}}\left(\alpha+\alpha^{q_{0}}\right) \\
& =\operatorname{Tr}_{\mathbb{F}_{q_{0}} / \mathbb{F}_{2}}(\alpha)+\operatorname{Tr}_{\mathbb{F}_{q_{0}} / \mathbb{F}_{2}}\left(\alpha^{q_{0}}\right) \\
& =0
\end{aligned}
$$

where the first and second equality come from the properties of the trace function and the last one from the fact that $\alpha \in \mathbb{F}_{q_{0}}$. Since $\mathbb{F}_{q_{0}}^{*}$ is a multiplicative group, the second part follows.

Proposition 2. Let $q_{0}=2^{m}$ and $q=q_{0}^{2}$. Then there exists a $\left[2 q_{0}, q_{0}, d \geq q_{0}\right]$ self-dual code over $\mathbb{F}_{q}$.

Proof. Consider the elliptic curve defined by

$$
\mathcal{E}_{1,1,0}: y^{2}+y=x^{3}+x
$$

From Lemma 13, we get that $\mathbb{F}_{q_{0}}$ is a subset of $S_{1,1,0}$. Put $U=\mathbb{F}_{q_{0}}$ and $h(x)=\prod_{\alpha \in U}(x-\alpha)$. Then the residue $\operatorname{Res}_{P_{\alpha}}(\omega)=\frac{1}{h^{\prime}\left(P_{\alpha}\right)}$ is a square for any $\alpha \in U$, and by Lemma 3 , the constructed code $a \cdot C_{\mathcal{L}}(D, G)$ is self-dual, where $a_{i}^{2}=\operatorname{Res}_{P_{i}}(\omega)$.

Theorem 5. Let $q=2^{m}$ and $U=\left\{\alpha \in \mathbb{F}_{q} \mid \operatorname{Tr}\left(\alpha^{3}+\alpha\right)=0\right\}$. Then there exists a self-dual code over $\mathbb{F}_{q}$ with parameters $[2 n, n, d \geq n]$ for $1 \leq n \leq|U|$.

Proof. Let $U$ be defined as in the theorem. Put

$$
h(x)=\prod_{\alpha \in U}(x-\alpha) .
$$

Since any element in $\mathbb{F}_{q}$ ( $q$ even) is a square in $\mathbb{F}_{q}$, we conclude that $h^{\prime}(\alpha)$ is a nonzero square in $\mathbb{F}_{q}$ for any $\alpha \in U$.

Consider the elliptic curve defined by

$$
\mathcal{E}_{1,1,0}: y^{2}+y=x^{3}+x
$$


From Lemma 12, we get that $U$ is a subset of $S_{1,1,0}$. Put

$$
D=\sum_{\alpha \in U_{0} \subset U}\left(P_{\alpha}^{(1)}+P_{\alpha}^{(2)}\right)=P_{1}+\cdots+P_{s}, s=2\left|U_{0}\right|, G=\frac{s}{2} P_{\infty}, \omega=\frac{d x}{h} .
$$

Then the residue $\operatorname{Res}_{P_{\alpha}}(\omega)=\frac{1}{h^{\prime}\left(P_{\alpha}\right)}$ is a square for any $\alpha \in U_{0}$, and by Lemma 3, the constructed code $a \cdot C_{\mathcal{L}}(D, G)$ is self-dual, where $a_{i}^{2}=\operatorname{Res}_{P_{i}}(\omega)$.

Example 3. The elliptic curve

$$
\mathcal{E}_{1,1,0}: y^{2}+y=x^{3}+x
$$

has rational points in the set $\left\{P_{\infty}=(1: 0: 0),(1: 0: 1),(1: 1: 1),\left(w^{3}\right.\right.$ : $\left.w^{7}: 1\right),\left(w^{3}: w^{9}: 1\right),\left(w^{6}: w^{3}: 1\right),\left(w^{6}: w^{14}: 1\right),\left(w^{12}: w^{6}: 1\right),\left(w^{12}:\right.$ $\left.w^{13}: 1\right),\left(w^{10}: w: 1\right),\left(w^{10}: w^{4}: 1\right),\left(w^{11}: w: 1\right),\left(w^{11}: w^{4}: 1\right),\left(w^{5}: w^{2}:\right.$ 1), $\left.\left(w^{5}: w^{8}: 1\right),\left(w^{7}: w^{2}: 1\right),\left(w^{7}: w^{8}: 1\right),\left(w^{13}: w^{2}: 1\right),\left(w^{13}: w^{8}: 1\right)\right\}$. Put $D=P_{1}+\cdots+P_{18}, G=9 P_{\infty}$. The code $C_{\mathcal{L}}(D, G)$ is self-dual. The set $\left\{\frac{x^{i} y^{j}}{z^{i+j}} \mid(i, j) \in\{(0,0),(0,1),(0,2),(0,3),(1,0),(1,1),(1,2),(2,0),(2,1)\}\right\}$ is a basis for $C_{\mathcal{L}}(D, G)$, and thus its generator matrix is given by

$$
\mathcal{G}=\left(\begin{array}{c}
11111111111111111 \\
01 w^{7} w^{9} w^{3} w^{14} w^{6} w^{13} w w^{4} w w^{4} w^{2} w^{8} w^{2} w^{8} w^{2} w^{8} \\
01 w^{14} w^{3} w^{6} w^{13} w^{12} w^{11} w^{2} w^{8} w^{2} w^{8} w^{4} w w^{4} w w^{4} w \\
01 w^{6} w^{12} w^{9} w^{12} w^{3} w^{9} w^{3} w^{12} w^{3} w^{12} w^{6} w^{9} w^{6} w^{9} w^{6} w^{9} \\
11 w^{3} w^{3} w^{6} w^{6} w^{12} w^{12} w^{10} w^{10} w^{11} w^{11} w^{5} w^{5} w^{7} w^{7} w^{13} w^{13} \\
01 w^{10} w^{12} w^{9} w^{5} w^{3} w^{10} w^{11} w^{14} w^{12} 1 w^{7} w^{13} w^{9} 11 w^{6} \\
01 w^{2} w^{6} w^{12} w^{4} w^{9} w^{8} w^{12} w^{3} w^{13} w^{4} w^{9} w^{6} w^{11} w^{8} w^{2} w^{14} \\
11 w^{6} w^{6} w^{12} w^{12} w^{9} w^{9} w^{5} w^{5} w^{7} w^{7} w^{10} w^{10} w^{14} w^{14} w^{11} w^{11} \\
01 w^{13} 11 w^{11} 1 w^{7} w^{6} w^{9} w^{8} w^{11} w^{12} w^{3} w w^{7} w^{13} w^{4}
\end{array}\right)
$$

By Magma [3], the code with generator matrix a. $\mathcal{G}$ is self-dual, and it has parameters $[18,9,9]$ over $\mathbb{F}_{16}$, where $a=\left(w^{5}, w^{5}, w^{12}, w^{12}, w^{7}, w^{7}, 1,1, w^{2}, w^{2}\right.$, $\left.w^{14}, w^{14}, w^{4}, w^{4}, w^{9}, w^{9}, w^{10}, w^{10}\right)$.

This code is an almost MDS code. We also find almost MDS self-dual codes over $\mathbb{F}_{16}$ with parameters $[20,10,10]$, [22, 11, 11], [24, 12, 12].

Corollary 1. Let $q=2^{m}$ and $U=\left\{\alpha \in \mathbb{F}_{q} \mid \operatorname{Tr}\left(\alpha^{3}\right)=0\right\}$. Then there exists a self-dual code over $\mathbb{F}_{q}$ with parameters $[2 n, n, d \geq n]$ for $1 \leq n \leq|U|$.

Theorem 6. Let $q=2^{m}, m \geq 3$ and $U=\left\{\alpha \in \mathbb{F}_{q} \mid \operatorname{Tr}\left(\alpha^{5}\right)=0\right\}$. Then there exists a self-dual code over $\mathbb{F}_{q}$ with parameters $[2 n, n, d \geq n-1]$ for $1 \leq n \leq|U|$. 
Proof. Let $U$ be defined as in the theorem. Put

$$
h(x)=\prod_{\alpha \in U}(x-\alpha) .
$$

Since any element in $\mathbb{F}_{q}$ ( $q$ even) is a square in $\mathbb{F}_{q}$, we conclude that $h^{\prime}(\alpha)$ is a nonzero square in $\mathbb{F}_{q}$ for any $\alpha \in U$.

Consider the hyper-elliptic curve defined by

$$
\mathcal{X}: y^{2}+y=x^{5} .
$$

From Lemma 12, we get that $U$ is a subset of the solution to (8). Put $D=\sum_{\alpha \in U_{0} \subset U}\left(P_{\alpha}^{(1)}+P_{\alpha}^{(2)}\right)=P_{1}+\cdots+P_{s}, s=2\left|U_{0}\right|, G=\left(\frac{s}{2}+1\right) P_{\infty}$ and $\omega=\frac{d x}{h}$. Then the residue $\operatorname{Res}_{P_{\alpha}}(\omega)=\frac{1}{h^{\prime}\left(P_{\alpha}\right)}$ is a square for any $\alpha \in U_{0}$, and by Lemma 3 , the constructed code $a \cdot C_{\mathcal{L}}(D, G)$ is a $\left[s, \frac{s}{2}, d \geq \frac{s}{2}-1\right]$ self-dual code, where $a_{i}^{2}=\operatorname{Res}_{P_{i}}(\omega)$.

Example 4. The hyper-elliptic curve defined by

$$
y^{2}+y=x^{5}
$$

has rational points in the set $\left\{P_{\infty}=(1: 0: 0),\left(w^{12}: 0: 1\right),\left(w^{12}: 1: 1\right),\left(w^{3}\right.\right.$ : $0: 1),\left(w^{3}: 1: 1\right),\left(w^{6}: 0: 1\right),\left(w^{6}: 1: 1\right),\left(w^{9}: 0: 1\right),\left(w^{9}: 1: 1\right),\left(w^{11}:\right.$ $w: 1),\left(w^{11}: w^{4}: 1\right),\left(w^{2}: w: 1\right),\left(w^{2}: w^{4}: 1\right),\left(w^{5}: w: 1\right),\left(w^{5}: w^{4}:\right.$ $1),\left(w^{8}: w: 1\right),\left(w^{8}: w^{4}: 1\right),\left(w: w^{2}: 1\right),\left(w: w^{8}: 1\right),\left(w^{13}: w^{2}: 1\right),\left(w^{13}:\right.$ $\left.w^{8}: 1\right),\left(w^{4}: w^{2}: 1\right),\left(w^{4}: w^{8}: 1\right),\left(w^{7}: w^{2}: 1\right),\left(w^{7}: w^{8}: 1\right),\left(w^{10}: w^{2}:\right.$ 1), $\left.\left(w^{10}: w^{8}: 1\right)\right\}$. Put $D=P_{1}+\cdots+P_{26}, G=14 P_{\infty}$. The set $\left\{\frac{x^{i} y^{j}}{z^{i+j}} \mid(i, j) \in\right.$ $\{(0,0),(0,1),(0,2),(1,0),(1,1),(1,2),(2,0),(2,1),(2,2),(3,0),(3,1),(4,0),(4,1)\}\}$ is a basis for $C_{\mathcal{L}}(D, G)$, and thus its generator matrix $\mathcal{G}$ is given by 


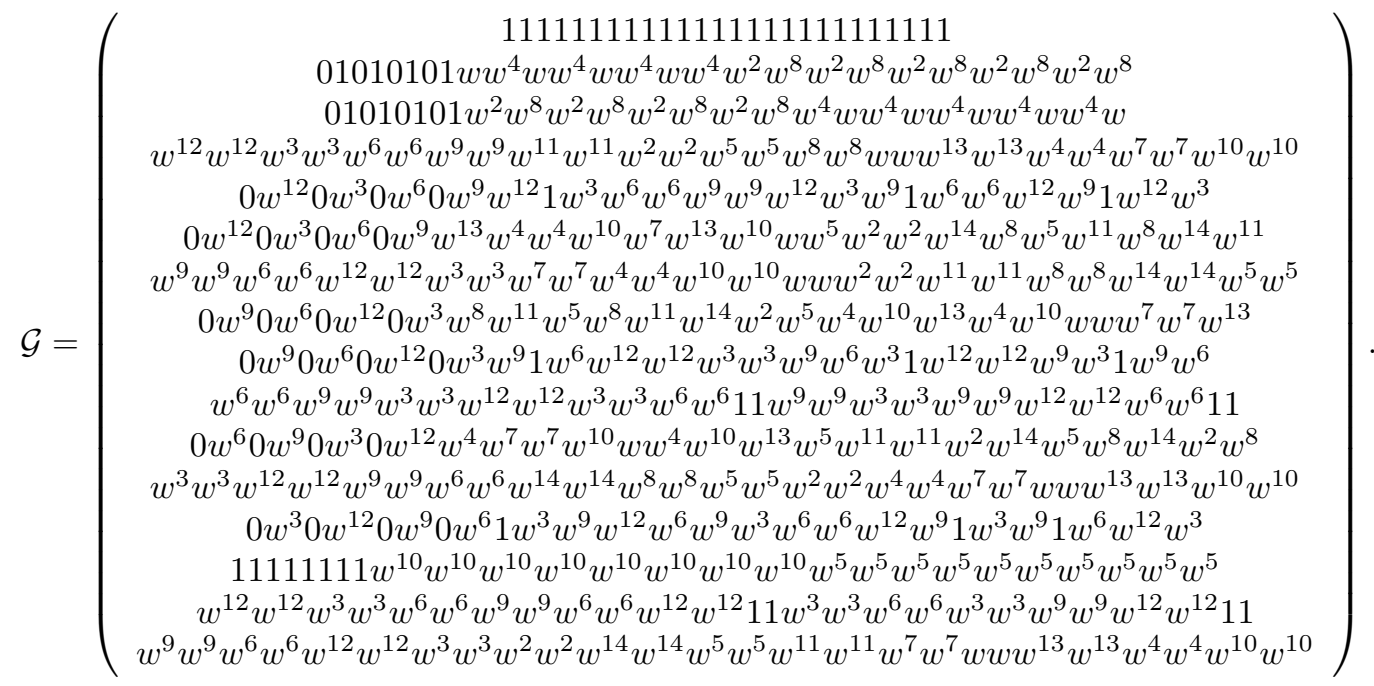

By Magma [3], the code with generator matrix a.G is self-dual, and it has parameters $[26,13,12]$ over $\mathbb{F}_{16}$, where $a=\left(w^{14}, w^{14}, w, w, w^{6}, w^{6}, w^{10}, w^{10}, w^{9}\right.$, $\left.w^{9}, w^{4}, w^{4}, w^{6}, w^{6}, w^{8}, w^{8}, w^{6}, w^{6}, w^{3}, w^{3}, w^{7}, w^{7}, w, w, w^{13}, w^{13}\right)$. We also find self-dual codes over $\mathbb{F}_{16}$ with parameters $[28,14,13]$, [30, 15, 14], [32, 16, 15].

Next, we will consider hyper-elliptic curves over $\mathbb{F}_{q}, q=p^{m}$ with $p$ an odd prime.

Theorem 7. Let $q=p^{m}$ and $t$ be a positive odd integer such that $g c d(t, q-$ $1)=1$. If $\eta(n)=1$ and $4 n \mid(q-1)$, then there exists a self-dual code with parameters $\left[2 n, n, d \geq n+\frac{t-3}{2}\right]$.

Proof. Denote $C_{j}=\{j \times i(\bmod q-1) \mid i=0,1, \ldots\}$. For $\theta$ a primitive element of $\mathbb{F}_{q}$, let $U_{n}=\left\{\theta^{i} \mid i \in C_{\frac{q-1}{n}}\right\}$, and label the elements of $U_{n}$ as $\alpha_{1}, \ldots, \alpha_{n}$. Under the condition $4 n \mid(q-1)$, the set $U_{n}$ is a multiplicative subgroup of $\mathbb{F}_{q}^{*}$ of order $n$. Put

$$
h(x)=\prod_{\alpha \in U_{n}}(x-\alpha) .
$$

Clearly, all the roots of $h(x)$ are simple, and the derivative $h^{\prime}(x)=n x^{n-1}$, and thus for any $\alpha \in U_{n}$, we have that $h^{\prime}(\alpha)$ is a square. Consider the elliptic curve defined by

$$
\mathcal{X}: y^{2}=x^{t}
$$


Since $\operatorname{gcd}(t, q-1)=1$, the set $\left\{x^{t} \mid x \in \mathbb{F}_{q}\right\}$ is in bijection with $\mathbb{F}_{q}$. For any $\alpha \in U_{n}$, there are two places, say $P_{\alpha}^{(1)}$ and $P_{\alpha}^{(2)}$, arising from $x$-component $\alpha$. Put $D=\sum_{\alpha \in U_{n}} P_{\alpha}^{(1)}+P_{\alpha}^{(2)}=P_{1}+\cdots+P_{s}, s=2 n, G=n P_{\infty}$ and $\omega=\frac{d x}{h}$. With the choice of $\alpha_{i} \in U_{n}$ and $\beta_{i}^{2}=\alpha_{i}^{t}$, the residue $\operatorname{Res}_{P_{\alpha_{i}}}(\omega)=\frac{1}{\beta_{i} h^{\prime}\left(\alpha_{i}\right)}$ is a square for any $\alpha_{i} \in U_{n}$, and by Lemma 3 , the constructed code $a \cdot C_{\mathcal{L}}(D, G)$ is self-dual, where $a_{i}^{2}=\operatorname{Res}_{P_{i}}(\omega)$.

Corollary 2. Let $q=p^{m}$. Then we have the following:

1. if $\operatorname{gcd}(3, q-1)=1, \eta(n)=1$ and $4 n \mid(q-1)$, then there exists a self-dual code with parameters $[2 n, n, d \geq n]$;

2. If $\operatorname{gcd}(5, q-1)=1, \eta(n)=1$ and $4 n \mid(q-1)$, then there exists a self-dual code with parameters $[2 n, n, d \geq n-1]$.

Example 5. The hyper-elliptic curve over $\mathbb{F}_{25}$ defined by

$$
y^{2}=x^{5}
$$

has rational points in the set $\left\{P_{\infty}=(1: 0: 0),(1: 1: 1),(1: 4\right.$ : $1),\left(w^{20}: w^{2}: 1\right),\left(w^{20}: w^{14}: 1\right),\left(w^{16}: w^{4}: 1\right),\left(w^{16}: w^{16}: 1\right),(4:$ $2: 1),(4: 3: 1),\left(w^{8}: w^{8}: 1\right),\left(w^{8}: w^{20}: 1\right),\left(w^{4}: w^{10}: 1\right),\left(w^{4}:\right.$ $\left.\left.w^{22}\right)\right\}$. Put $D=P_{1}+\cdots+P_{12}$ and $G=7 P_{\infty}$. The set $\left\{\frac{x^{i} y^{j}}{z^{i+j}} \mid(i, j) \in\right.$ $\{(0,0),(0,1),(1,0),(1,1),(2,0),(3,0)\}\}$ is a basis for $C_{\mathcal{L}}(D, G)$, and thus its generator matrix is given by

$$
\mathcal{G}=\left(\begin{array}{cccccccccccc}
1 & 1 & 1 & 1 & 1 & 1 & 1 & 1 & 1 & 1 & 1 & 1 \\
1 & 4 & w^{2} & w^{14} & w^{4} & w^{16} & 2 & 3 & w^{8} & w^{20} & w^{10} & w^{22} \\
1 & 1 & w^{20} & w^{20} & w^{16} & w^{16} & 4 & 4 & w^{8} & w^{8} & w^{4} & w^{4} \\
1 & 4 & w^{22} & w^{10} & w^{20} & w^{8} & 3 & 2 & w^{16} & w^{4} & w^{14} & w^{2} \\
1 & 1 & w^{16} & w^{16} & w^{8} & w^{8} & 1 & 1 & w^{16} & w^{16} & w^{8} & w^{8} \\
1 & 1 & 4 & 4 & 1 & 1 & 4 & 4 & 1 & 1 & 4 & 4
\end{array}\right)
$$

By Magma [3], the code with generator matrix $\cdot \mathcal{G}$ is self-dual, and it has parameters $[12,6,5]$ over $\mathbb{F}_{25}$, where $a=\left(1,3, w^{21}, w^{15}, 3,1, w^{15}, w^{21}, 1,3, w^{21}, w^{15}\right)$.

By considering curves in higher genus, we can release the gcd condition in Theorem 7 . 
Theorem 8. Let $q=p^{m}$ with $p$ an odd prime.

1. If $n$ is odd, $\eta(n)=1$ and $4 n \mid(q-1)$, then there exists a self-dual code with parameters $\left[2 n, n, d \geq \frac{n}{2}+2\right]$.

2. If $n$ is even, $\eta(n)=1$ and $2 n \mid(q-1)$, then there exists a self-dual code with parameters $\left[2 n, n, d \geq \frac{n}{2}+2\right]$.

Proof. Assume that $n$ is odd. Let $U_{n}$ and $h(x)$ be defined as in Theorem 7 . Consider an algebraic curve given by

$$
\mathcal{X}: y^{2}=x^{n} .
$$

Take $\omega=\frac{d x}{h}$ and $G=\frac{3 n-4}{2} P_{\infty}$. Then by Lemma 3, the code $a \cdot C_{\mathcal{L}}(D, G)$, where $a_{i}=\operatorname{Res}_{P_{i}}(\omega)$, is self-dual with parameters $\left[2 n, n, \frac{n}{2}+2\right]$, and this proves point 1).

For point 2), we put $U_{n}=\left\{a^{2} \mid a \in \mathbb{F}_{q}, a^{n}=1\right\}$. The rest follows from the same reasoning as the first part.

\subsection{Self-dual codes from other curves}

In this subsection, we will construct self-dual codes over $\mathbb{F}_{q}$ from algebraic curves of high genus.

Let $q_{0}=p^{m}, q=q_{0}^{2}$ and $\mathcal{X}$ be the Hermitian curve over $\mathbb{F}_{q}$ defined by

$$
\mathcal{X}: y^{q_{0}}+y=x^{q_{0}+1} .
$$

The Hermitian curve $\mathcal{X}$ has genus $g=\frac{q_{0}\left(q_{0}-1\right)}{2}$, and for any $\alpha \in \mathbb{F}_{q}, x-\alpha$ has $q$ zeros of degree one in $\mathcal{X}$. All rational points of the curve $\mathcal{X}$ different from the point at infinity are obtained in this way. Self-orthogonal AG codes from Hermitian curves were already considered in [20. In what follows, we embed those codes into the self-dual ones and provide the parameters of the latter codes. We also construct new families of self-dual codes from this curve.

Theorem 9. Let $p$ be an odd prime, $q_{0}=p^{m}, q=q_{0}^{2}, g=\frac{q_{0}\left(q_{0}-1\right)}{2}$. Put $d_{0}=\frac{s}{2}+1-g, s^{\prime}=s+1, d_{0}^{\prime}=\frac{s^{\prime}}{2}-g$.

1. If $p|n,(n-1)|(q-1)$, then there exists a q-ary self-dual code with parameters $\left[s, \frac{s}{2}, d \geq d_{0}\right]$ (resp. $\left.\left[s^{\prime}, \frac{s^{\prime}}{2}, d \geq d_{0}^{\prime}\right]\right)$, where $s=q_{0} n$ with $n$ even (resp. $n$ odd). 
2. If $r \mid m$, then there exists a q-ary self-dual code with parameters $\left[s^{\prime}, \frac{s^{\prime}}{2}, d \geq\right.$ $\left.d_{0}^{\prime}\right]$, where $s=q_{0} p^{r}$.

3. If $(n-1) \mid(q-1)$, then there exists a q-ary self-dual code with parameters $\left[s^{\prime}, \frac{s^{\prime}}{2}, d \geq d_{0}^{\prime}\right]$, where $s=q_{0}(2 n-1)$.

4. If $n \mid \frac{q-1}{2}$, then there exists a q-ary self-dual code with parameters $\left[s, \frac{s}{2}, d \geq\right.$ $\left.d_{0}\right]$ (resp. $\left[s^{\prime}, \frac{s^{\prime}}{2}, d \geq d_{0}^{\prime}\right]$ ), where $s=q_{0} n$ with $n$ even (resp. $n$ odd).

5. If $1 \leq r<m$, then there exists a q-ary self-dual code with parameters $\left[s^{\prime}, \frac{s^{\prime}}{2}, d \geq d_{0}^{\prime}\right]$, where $s=q_{0}\left(2 p^{r}-1\right)$.

6. If $n=q_{0}-1, n \equiv 0(\bmod 4)$, then there exists a q-ary self-dual code with parameters $\left[s^{\prime}, \frac{s^{\prime}}{2}, d \geq d_{0}^{\prime}\right]$, where $s=q_{0}(n(t+1)+1)$, for $t$ odd, $0 \leq t \leq \frac{n}{2}+1$.

7. If $n=q_{0}-1, n \equiv 2(\bmod 4)$, then there exists a q-ary self-dual code with parameters $\left[s^{\prime}, \frac{s^{\prime}}{2}, d \geq d_{0}^{\prime}\right]$, where $s=q_{0}(n(t+1)+1), 0 \leq t \leq \frac{n}{2}$.

8. If $1 \leq r<m$ and $\frac{n\left(p^{r}+1\right)}{2\left(p^{r}-1\right)}$ is odd, then there exists a q-ary self-dual code with parameters $\left[s, \frac{s}{2}, d \geq d_{0}\right]$ (resp. $\left.\left[s^{\prime}, \frac{s^{\prime}}{2}, d \geq d_{0}^{\prime}\right]\right)$, where $s=q_{0}(t+1) n$ (resp. $\left.s=q_{0}((t+1) n+1)+1\right), n=\frac{q-1}{p^{r}+1}$, for $t$ odd, $1 \leq t \leq p^{r}$.

9. If $1 \leq r<m$ and $\frac{n\left(p^{r}+1\right)}{2\left(p^{r}-1\right)}$ is even, then there exists a $q$-ary self-dual code with parameters $\left[s, \frac{s}{2}, d \geq d_{0}\right]$ (resp. $\left.\left[s^{\prime}, \frac{s^{\prime}}{2}, d \geq d_{0}^{\prime}\right]\right)$, where $s=q_{0}(t+1) n$ (resp. $\left.s=q_{0}((t+1) n+1)+1\right), n=\frac{q-1}{p^{r}+1}$, for $1 \leq t \leq p^{r}$.

10. If $1 \leq r<m$, then there exists a q-ary self-dual code with parameters $\left[s, \frac{s}{2}, d \geq d_{0}\right]$ (resp. $\left[s^{\prime}, \frac{s^{\prime}}{2}, d \geq d_{0}^{\prime}\right]$ ), where $s=q_{0}(t+1) n$ (resp. $s=$ $\left.q_{0}((t+1) n+1)+1\right), n=\frac{q-1}{p^{r}-1}, r \mid \frac{m}{2}$, for $1 \leq t \leq p^{r}-2$.

11. If $t$ is even such that $1 \leq t \leq q_{0}$, then there exists a $q$-ary self-dual code with parameters $\left[s, \frac{s}{2}, d \geq d_{0}\right]$, where $s=q_{0}\left(q_{0} t\right)$.

12. If $t$ is odd such that $1 \leq t \leq q_{0}$, then there exists a q-ary self-dual code with parameters $\left[s^{\prime}, \frac{s^{\prime}}{2}, d \geq d_{0}^{\prime}\right]$, where $s=q_{0}\left(q_{0} t\right)$.

13. If $r=p^{k}, k \mid m, 0 \leq \ell<m / k, 1 \leq t \leq(r-1) / 2$, then there exists a $q$-ary self-dual code with parameters $\left[s, \frac{s}{2}, d \geq d_{0}\right]$, where $s=q_{0}\left(2 t r^{\ell}\right)$. 
14. If $0 \leq \ell<2 m$, then there exists a q-ary self-dual code with parameters $\left[s, \frac{s}{2}, d \geq d_{0}\right]$, where $s=q_{0}\left(2 p^{\ell}\right)$.

15. If $r=p^{k}, k \mid m, 0 \leq \ell<m / k, 0 \leq t \leq(r-1) / 2$ or $(\ell, t)=(m / k, 0)$, then there exists a q-ary self-dual code with parameters $\left[s^{\prime}, \frac{s^{\prime}}{2}, d \geq d_{0}^{\prime}\right]$, where $s=q_{0}(2 t+1) r^{\ell}$.

16. If $0 \leq \ell<2 m$, then there exists a q-ary self-dual code with parameters $\left[s^{\prime}, \frac{s^{\prime}}{2}, d \geq d_{0}^{\prime}\right]$, where $s=q_{0} p^{\ell}$.

Proof. It should be noted that each $x$-component $\alpha \in \mathbb{F}_{q}$ gives $q$ places of degree one. Let $U$ be a subset of $\left\{\alpha \in \mathbb{F}_{q} \mid \beta^{q_{0}}+\beta=\alpha^{q_{0}+1}\right\}$ such that $q|U|=s$. Put

$$
h(x)=\prod_{\alpha \in U}(x-\alpha) \text { and } \omega=\frac{d x}{h} .
$$

For each case, it is enough to prove that the residue $\operatorname{Res}_{P_{\alpha}}(\omega)$ of $\omega$ at place $P_{\alpha}$ is a nonzero square for any $\alpha \in U$, that is, $h^{\prime}(\alpha)$ is a nonzero square in $\mathbb{F}_{q}$. Take $U$ as follows.

- for 1), $U=\left\{\alpha \in \mathbb{F}_{q} \mid \alpha^{n}=\alpha\right\}$,

- for 2), $U=\left\{\alpha \in \mathbb{F}_{q} \mid \alpha^{p^{r}}=\alpha\right\}$,

- for 3), $U=U_{n-1} \cup \alpha_{1} U_{n-1}$, where $U_{n-1}=\left\{\alpha \in \mathbb{F}_{q} \mid \alpha^{n-1}=1\right\}$ and $\alpha_{1} \in \mathbb{F}_{q} \backslash U_{n-1}$ such that $1-\alpha_{1}^{n-1}$ is a square,

- for 4$), U=\left\{\alpha \in \mathbb{F}_{q} \mid \alpha^{n}=1\right\}$,

- for 5), $U=U_{n} \cup \alpha_{1} U_{n} \cup\{0\}$, where $n=p^{r}-1, U_{n}=\left\{\alpha \in \mathbb{F}_{q} \mid \alpha^{n}=1\right\}$ and $\alpha_{1} \in \mathbb{F}_{q} \backslash U_{n}$ such that $1-\alpha^{n}$ is a square,

- for 6)-10), take $U=\{0\} \cup V$ or $U=V$, where $V=U_{n} \cup \alpha_{1} U_{n} \cup \cdots \cup$ $\alpha_{t} U_{n}, U_{n}=\left\{\alpha \in \mathbb{F}_{q} \mid \alpha^{n}=1\right\}$ and $\alpha_{1}, \ldots, \alpha_{t} \in \mathbb{F}_{q} \backslash U_{n}$ as in Theorem 3 ,

- for 10)-12), label the elements of $\mathbb{F}_{q_{0}}$ as $a_{1}, \ldots, a_{q_{0}}$. For some fixed element $\beta \in \mathbb{F}_{q} \backslash \mathbb{F}_{q_{0}}$, take $U=\left\{a_{k} \beta+a_{j} \mid 1 \leq k, j \leq q_{0}\right\}$,

- for 13)-16), label the element of $\mathbb{F}_{r}$ as $a_{0}, \ldots, a_{r-1}$, take $H$ as an $\mathbb{F}_{r^{-}}$ subspace and set $H_{i}=H+a_{i} \beta$ for some fixed element $\beta \in \mathbb{F}_{q} \backslash \mathbb{F}_{r}$. Put $U=H_{0} \cup \cdots \cup H_{2 t-1}$ or $U=H_{0} \cup \cdots \cup H_{2 t}$. 
For 1)-5), it can be easily checked that $h^{\prime}(\alpha)$ is a square for any $\alpha \in U$.

For 6$)-10)$, it has been already checked, in Theorem 3 , that $h^{\prime}(\alpha)$ is a square for any $\alpha \in U$.

For 11)-12), it was proved in [24, Theorem 2] that $h^{\prime}(\alpha)$ is a square for any $\alpha \in U$.

For 13)-16), it was proved in [7, Theorem 4] that $h^{\prime}(\alpha)$ is a square for any $\alpha \in U$.

Example 6. The Hermitian curve defined over $\mathbb{F}_{9}$ has all rational points in the set $\left\{P_{\infty}=(1: 0: 0),(0: 0: 1),\left(0: w^{2}: 1\right),\left(0: w^{6}: 1\right),(1: w: 1),(1:\right.$ $\left.w^{3}: 1\right),(1: 2: 1),\left(w^{2}: w: 1\right),\left(w^{2}: w^{3}: 1\right),\left(w^{2}: 2: 1\right),(2: w: 1),(2:$ $\left.w^{3}: 1\right),(2: 2: 1),\left(w^{6}: w: 1\right),\left(w^{6}: w^{3}: 1\right),\left(w^{6}: 2: 1\right),(w: 1: 1),(w:$ $\left.w^{5}: 1\right),\left(w: w^{7}: 1\right),\left(w^{3}: 1: 1\right),\left(w^{3}: w^{5}: 1\right),\left(w^{3}: w^{7}: 1\right),\left(w^{5}: 1: 1\right),\left(w^{5}:\right.$ $\left.\left.w^{5}: 1\right),\left(w^{5}: w^{7}: 1\right),\left(w^{7}: 1: 1\right),\left(w^{7}: w^{5}: 1\right),\left(w^{7}: w^{7}: 1\right)\right\}$. Put $D=P_{1}+$ $\cdots+P_{27}, G=15 P_{\infty}$. The code $C_{\mathcal{L}}(D, G)$ has parameters $[27,13,12]$. The set $\left\{\frac{x^{i} y^{j}}{z^{i+j}} \mid(i, j) \in\{(0,0),(0,1),(0,2),(0,3),(1,0),(1,1),(1,2),(1,3),(2,0),(2,1)\right.$, $(2,2),(3,0),(3,1)\}\}$ is a basis for the code $C_{\mathcal{L}}(D, G)$, and thus its generator matrix is given by

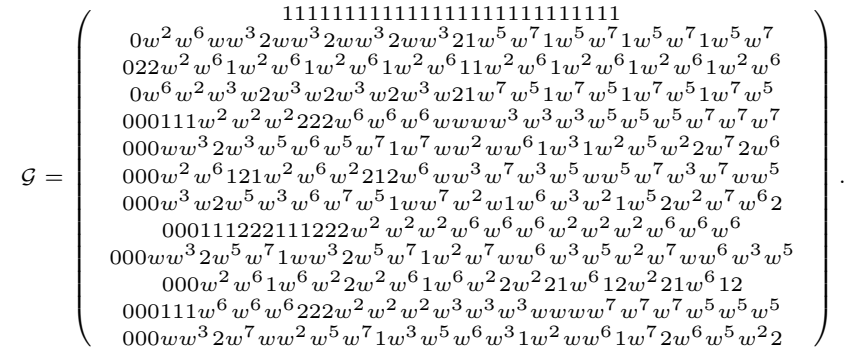

Take $\mathcal{G}^{\prime}=\left(\begin{array}{c}\mathcal{G} 0 \\ g_{14}\end{array}\right)$, where $g_{14}=(0,1,1,2,2,1,2,2,1,2,2,1,2,2,1,1,2,2$, $1,2,2,1,2,2,1,2,2,1)$. By Magma [3], the code with generator matrix a $\cdot \mathcal{G}^{\prime}$ is an optimal $[28,14,12]$ self-dual code with new parameters, where $a_{i}=w^{6}, 1 \leq$ $i \leq 28$. Other parameters from different constructions in Theorem 9 are given in Table 3 .

Theorem 10. Let $q_{0}=p^{m}, q=q_{0}^{2}$ be an odd prime power, $g=\frac{\left(q_{0}-1\right)^{2}}{4}$ and $s=q_{0} \frac{q_{0}^{2}+1}{2}$. Then,

1. there exists $a\left[s, \frac{s}{2}, d \geq \frac{s}{2}-g+1\right]$ self-dual code if $s$ is even, and

2. there exists $a\left[s+1, \frac{s+1}{2}, d \geq \frac{s+1}{2}-g\right]$ self-dual code if $s$ is odd. 
Table 3: Self-dual codes of length $s$ from Hermitian curves defined over $\mathbb{F}_{9}$

\begin{tabular}{cccc|ccc} 
Theorem 9 & Length $s$ & Distance & Lower bound & Extended length & Distance & Lower bound \\
\hline \multirow{2}{*}{$1)$} & 3.3 & 3 & 3 & 10 & 3 & 12 \\
& 3.9 & 12 & 12 & 28 & 3 & 2 \\
\hline 2$)$ & 3.3 & 3 & 3 & 10 & 6 & - \\
\hline 3$)$ & $3(2.3-1)$ & 6 & 6 & - & - & - \\
\hline 4$)$ & 3.2 & 3 & 4 & 4 & - & - \\
\hline 5$)$ & $3(1+1) 2$ & 4 & 4 & 16 & 6 & - \\
\hline 6$)$ & $3((1+1) 2+1)$ & 6 & 6 & - & -
\end{tabular}

Proof. Consider an algebraic curve defined by

$$
\mathcal{X}: y^{q_{0}}+y=x^{\frac{q_{0}+1}{2}} .
$$

The curve has genus $g=\frac{\left(q_{0}-1\right)^{2}}{4}$. Put

$$
U=\left\{\alpha \in \mathbb{F}_{q} \mid \exists \beta \in \mathbb{F}_{q} \text { such that } \beta^{q_{0}}+\beta=\alpha^{\frac{q_{0}+1}{2}}\right\} .
$$

The set $U$ is the set of $x$-component solutions to the Hermitian curve whose elements are squares in $\mathbb{F}_{q}$. There are $\frac{q_{0}^{2}+1}{2}$ square elements in $\mathbb{F}_{q}$, and this gives rise to $q_{0} \frac{q_{0}^{2}+1}{2}$ rational places. Write

$$
h(x)=\prod_{\alpha \in U}(x-\alpha) \text { and } \omega=\frac{d x}{h} .
$$

Then $h(x)=x^{n}-x$, where $n=\frac{q_{0}^{2}+1}{2}$, and thus $h^{\prime}(x)=n x^{n-1}-1$. Since $q$ is a square, we have that $h^{\prime}(\alpha)=n-1$ is a square for any $\alpha \in U \backslash\{0\}$. Put $D=\sum_{\alpha \in U}\left(P_{\alpha}^{(1)}+\cdots+P_{\alpha}^{\left(q_{0}\right)}\right)=P_{1}+\cdots+P_{s}, s=q_{0} \frac{q_{0}^{2}+1}{2}$. Set

$$
G=\left\{\begin{array}{l}
\left(g-1+\frac{s}{2}\right) P_{\infty} \text { if } s \text { is even } \\
\left(g-1+\frac{s-1}{2}\right) P_{\infty} \text { if } s \text { is odd }
\end{array}\right.
$$

Then the residue $\operatorname{Res}_{P_{\alpha}}(\omega)=\frac{1}{h^{\prime}\left(P_{\alpha}\right)}$ is a square for any $\alpha \in U$, and by Lemma 3. the constructed code $a \cdot C_{\mathcal{L}}(D, G)$ is self-orthogonal, where $a_{i}^{2}=\operatorname{Res}_{P_{i}}(\omega)$. If $s$ is even, then point 1) follows, otherwise the self-orthogonal code can be embedded into a self-dual code using Lemma 6, and thus point 2) follows. 
Example 7. There exist self-dual codes with parameters $[16,8, \geq 7]_{3^{2}},[66,33, \geq$ $29]_{5^{2}},[176,88, \geq 79]_{7^{2}},[370,185, \geq 169]_{9^{2}},[672,336, \geq 311]_{11^{2}},[1106,553, \geq$ $517]_{13^{2}},[2466,1233, \geq 1169]_{17^{2}},[3440,1720, \geq 1639]_{19^{2}},[7826,3913, \geq 3769]_{25^{2}}$. We now calculate the exact distance of the self-dual code over $\mathbb{F}_{3^{2}}$. The algebraic curve over $\mathbb{F}_{9}$ defined by

$$
y^{3}+y=x^{2}
$$

has all rational points in the set $\left\{P_{\infty}=(1: 0: 0),(0: 0: 1),\left(0: w^{2}:\right.\right.$ $1),\left(0: w^{6}: 1\right),(1: w: 1),\left(1: w^{3}: 1\right),(1: 2: 1),(2: w: 1),\left(2: w^{3}: 1\right),(2: 2:$ $1),\left(w^{2}: 1: 1\right),\left(w^{2}: w^{5}: 1\right),\left(w^{2}: w^{7}: 1\right),\left(w^{6}: 1: 1\right),\left(w^{6}: w^{5}: 1\right),\left(w^{6}: w^{7}:\right.$ 1)\}. Put $D=P_{1}+\cdots+P_{15}, G=7 P_{\infty}$. The code $C_{\mathcal{L}}(D, G)$ has parameters $[15,7,8]$.

The set $\left\{\frac{x^{i} y^{j}}{z^{i+j}} \mid(i, j) \in\{(0,0),(0,1),(0,2),(0,3),(1,0),(1,1),(1,2)\}\right\}$ is a basis for the code $C_{\mathcal{L}}(D, G)$, and thus its generator matrix is given by

$$
\mathcal{G}=\left(\begin{array}{ccccccccccccccc}
1 & 1 & 1 & 1 & 1 & 1 & 1 & 1 & 1 & 1 & 1 & 1 & 1 & 1 & 1 \\
0 & w^{2} & w^{6} & w & w^{3} & 2 & w & w^{3} & 2 & 1 & w^{5} & w^{7} & 1 & w^{5} & w^{7} \\
0 & 2 & 2 & w^{2} & w^{6} & 1 & w^{2} & w^{6} & 1 & 1 & w^{2} & w^{6} & 1 & w^{2} & w^{6} \\
0 & w^{6} & w^{2} & w^{3} & w & 2 & w^{3} & w & 2 & 1 & w^{7} & w^{5} & 1 & w^{7} & w^{5} \\
0 & 0 & 0 & 1 & 1 & 1 & 2 & 2 & 2 & w^{2} & w^{2} & w^{2} & w^{6} & w^{6} & w^{6} \\
0 & 0 & 0 & w & w^{3} & 2 & w^{5} & w^{7} & 1 & w^{2} & w^{7} & w & w^{6} & w^{3} & w^{5} \\
0 & 0 & 0 & w^{2} & w^{6} & 1 & w^{6} & w^{2} & 2 & w^{2} & 2 & 1 & w^{6} & 1 & 2
\end{array}\right)
$$

Take

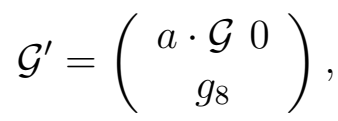

where $a=\left(w^{6}, w^{6}, w^{6}, 1,1,1,1,1,1,1,1,1,1,1,1\right), g_{8}=\left(0, w, 0,0,0,0,0,0, w^{7}\right.$, $\left.0, w^{5}, w^{2}, w^{5}, w^{7}, w^{7}, 2\right)$. By Magma [3], the code with generator matrix $\mathcal{G}^{\prime}$ is $a[16,8,8]$ self-dual code, which is optimal and has new parameters.

Theorem 11. Let $q_{0}=p^{m}, q=q_{0}^{2}$ be an odd prime power, $g=\frac{\left(q_{0}-1\right)^{2}}{4}$ and $s=q_{0} \frac{q_{0}^{2}-1}{2}$. Then,

1. there exists $a\left[s, \frac{s}{2}, d \geq \frac{s}{2}-g+1\right]$ self-dual code if $s$ is even, and

2. there exists $a\left[s+1, \frac{s+1}{2}, d \geq \frac{s+1}{2}-g\right]$ self-dual code if $s$ is odd. 
Proof. Consider the same setting as the proof of Theorem 10. Take $U^{\prime}=$ $U \backslash\{0\}$, and write

$$
h(x)=\prod_{\alpha \in U^{\prime}}(x-\alpha) \text { and } \omega=\frac{d x}{h} .
$$

The rest follows with the same reasoning as that in Theorem 10 .

Example 8. There exist self-dual codes with parameters $[12,6,6]_{3^{2}},[60,30, \geq$ $27]_{5^{2}},[168,84, \geq 76]_{7^{2}},[360,180, \geq 165]_{9^{2}},[660,330, \geq 306]_{11^{2}},[1092,546, \geq$ $511]_{13^{2}},[2448,1224, \geq 1161]_{17^{2}},[3420,1710, \geq 1630]_{19^{2}},[7800,3900, \geq 3757]_{25^{2}}$.

We update parameters of MDS self-dual codes from the previous constructions in Table 4

\section{Conclusion}

In this correspondence, we have constructed new families of optimal $q$-ary Euclidean self-dual codes from algebraic curves. With the same spirit, constructing more families of Euclidean self-dual codes from genus zero and genus one curves (over $\mathbb{F}_{q}$ with $q$ a prime) is worth considering. Characterization and constructions of Hermitian self-dual codes from algebraic geometry codes are also valuable.

\section{References}

[1] S. Ball, "On sets of vectors of a finite space in which every subset of basis size is a basis," J. Eur. Soc. 14 (2012), 733-748.

[2] K. Betsumiya, S. Georgiou, T. A. Gulliver, M. Harada and C. Koukouvinos, "On self-dual codes over some prime fields," Discrete Math. 262 (2003) 37-58.

[3] W. Bosma and J. Cannon, Handbook of Magma Functions, Sydney, 1995.

[4] R. Cramer, V. Daza, I. Gracia, J. J. Urroz, G. Leander, J. Marti-Farre and C. Padro, "On codes, matroids, and secure multiparty computation from linear secret-sharing schemes," IEEE Trans. Inform. Theory, vol. 54(6), pp. 2647-2657, 2008. 
Table 4: MDS self-dual codes of length $n$ over $\mathbb{F}_{q},-$ : no self-dual code exists with such a pair $(n, q)$, +: known parameters, ?: unknown parameters, *: new parameters

\begin{tabular}{c|c|c|c|c|c|c|c|c|c|c|c|c|c|c|c|c|c|c|c|c}
$n / q$ & 11 & 13 & 16 & 17 & 19 & 23 & 25 & 27 & 29 & 31 & 32 & 37 & 41 & 43 & 47 & 49 & 53 & 61 & 73 & 81 \\
\hline 2 & - & + & + & + & - & - & + & - & + & - & + & + & + & - & - & + & + & + & + & + \\
\hline 4 & + & + & + & + & + & + & + & + & + & + & + & + & + & + & + & + & + & + & + & + \\
\hline 6 & - & + & + & + & - & - & + & - & + & - & + & + & + & - & - & + & + & + & + & + \\
\hline 8 & + & + & + & + & + & + & + & + & + & + & + & + & + & + & + & + & + & + & + & + \\
\hline 10 & - & + & + & + & - & - & + & - & + & - & + & + & + & - & - & + & + & + & + & + \\
\hline 12 & + & 6 & + & + & + & + & + & + & + & + & + & + & + & + & + & + & + & + & + & + \\
\hline 14 & - & + & + & $?$ & - & - & + & - & + & - & + & + & + & - & - & + & + & + & + & + \\
\hline 16 & & & + & $?$ & $?$ & + & + & + & + & + & + & + & + & + & + & + & $?$ & + & $?$ & + \\
\hline 18 & & & & + & - & - & + & - & + & - & + & + & + & - & - & + & $?$ & $?$ & + & + \\
\hline 20 & & & & & + & $?$ & + & $?$ & $?$ & + & + & $?$ & + & + & + & + & $?$ & + & + & + \\
\hline 22 & - & & & & - & - & $?$ & - & $?$ & - & + & $?$ & + & $?$ & $?$ & + & $?$ & + & $?$ & + \\
\hline 24 & & & & & & + & $?$ & $?$ & $?$ & + & + & $?$ & $?$ & + & + & + & $?$ & $?$ & + & $*$ \\
\hline 26 & - & & & & - & - & + & - & $?$ & - & + & $*$ & $?$ & - & - & + & + & $*$ & + & + \\
\hline 28 & & & & & & & & + & $?$ & $?$ & + & $?$ & $?$ & $?$ & $?$ & + & $?$ & $?$ & $?$ & + \\
\hline 30 & - & & & & - & - & & & + & & + & $?$ & $?$ & - & - & $?$ & $?$ & + & $?$ & + \\
\hline 32 & & & & & & & & & & + & + & $?$ & $*$ & $?$ & $?$ & $?$ & $?$ & $?$ & $?$ & + \\
\hline 34 & - & & & & - & - & & - & & - & & $?$ & $?$ & - & - & + & $?$ & $?$ & $?$ & + \\
\hline 36 & & & & & & & & & & & & $?$ & $?$ & $?$ & $?$ & + & $?$ & $?$ & + & + \\
\hline 38 & - & & & & - & - & & - & & - & & + & $?$ & - & - & + & $?$ & $?$ & + & $?$ \\
\hline 40 & & & & & & & & & & & & & $?$ & $?$ & $?$ & $?$ & $?$ & $?$ & $?$ & + \\
\hline 42 & - & & & & - & - & & - & & - & & & + & - & - & + & $?$ & $*$ & $?$ & + \\
\hline 44 & & & & & & & & & & & & & & + & $?$ & $?$ & $?$ & $?$ & $?$ & $?$ \\
\hline 46 & - & & & & - & - & & - & & - & & & & - & - & $?$ & $?$ & $?$ & $?$ & + \\
\hline 48 & & & & & & & & & & & & & & & + & $?$ & $?$ & $?$ & $?$ & $?$ \\
\hline 50 & - & & & & - & - & & - & & - & & & & - & - & + & $?$ & $?$ & $*$ & + \\
\hline 52 & & & & & & & & & & & & & & & & & $?$ & $?$ & $?$ & + \\
\hline 62 & - & & & & - & - & & - & & - & & & & - & - & & & + & $?$ & + \\
\hline 72 & & & & & & & & & & & & & & & & & & $?$ & $?$ & +
\end{tabular}

[5] S. T. Dougherty, S. Mesnager, and P. Solé, "Secret-sharing schemes based on self-dual codes," in Proc. Inf. Theory Workshop, May 2008, pp. 338-342.

[6] Y. Driencourt and H. Stichtenoth, "A criterion for self-duality of geometric codes," Communications in Algebra, 1989, vol. 17(4), pp. 885-898.

[7] W. Fang and F. Fu, "New Constructions of MDS Euclidean Self-dual Codes from GRS Codes and Extended GRS Codes," IEEE Trans. Inform. Theory, vol. 65(9), pp. 5574-5579, 2019. 
[8] S. Georgiou and C. Koukouvinos, "MDS Self-Dual Codes over Large Prime Fields," Finite Fields and Their Applications, vol. 8, pp. 455470, 2002.

[9] V. D. Goppa, "Algebraico-geometric codes," Math. USSR-lvz. 21(1) (1983) 75-91.

[10] M. Grassl and T. A. Gulliver, "On Self-Dual MDS Codes" ISIT 2008, Toronto, Canada, July 6 -11, 2008.

[11] K. Guenda, "New MDS self-dual codes over finite fields," Des. Codes Cryptogr. (2012) 62:31-42.

[12] W. C. Huffman and V. Pless, Fundamentals of Error-Correcting Codes, Cambridge University Press, 2003.

[13] L. F. Jin and C. P. Xing, "New MDS self-dual codes from generalized Reed-Solomon codes," IEEE Trans. Inform. Theory, vol. 63(3), pp. $1434-1438,2017$.

[14] J-L. Kim and Y. Lee, "Construction of MDS self-dual codes over Galois rings," Des. Codes Cryptogr. (2007) 45:247-258.

[15] J-L. Kim and Y. Lee, "Euclidean and Hermitian self-dual MDS codes over large finite fields," J. Combin. Theory, Ser. A, vol. 105, pp. 79-95, 2004 .

[16] F. J. MacWilliams, N. J. A. Sloane and J.G. Thompson, "Good self-dual codes exist," Discrete Math. , vol. 3, pp. 153-162, 1972.

[17] F. J. MacWilliams and N. J. A. Sloane, The Theory of Error-Correcting Codes, Amsterdam, The Netherlands: North Holland, 1977.

[18] J. Massey, "Some applications of coding theory in cryptography," in Proc. 4th IMA Conf. Cryptogr. Coding, 1995, pp. 33-47.

[19] L. Sok, "Explicit constructions of MDS self-dual codes," IEEE Trans. Inform. Theory, DOI: 10.1109/TIT.2019.2954877.

[20] H. Stichtenoth, "Self-dual Goopa codes," Journal of Pure and Applied Algebra, 55 (1988), pp. 199-211. 
[21] H. Stichtenoth, "Algebraic function fields and codes," Springer, 2008.

[22] M.A. Tsfasman and S.G. Vladut, "Algebraic-Geometric Codes" Mathematics and Its Applications, Springer, 1991.

[23] H. Tong and X. Wang, "New MDS Euclidean and Hermitian self-dual codes over finite fields," Adv. in Pure Math. , vol. 7, pp. 325-333, May. 2017.

[24] H. Yan, "A note on the constructions of MDS self-dual codes," Cryptogr. Commun., (2019) 11:259-268, https://doi.org/10.1007/s12095018-0288-3. 